\title{
Inferring telescope polarization properties through spectral lines without linear polarization
}

\author{
A. Derks ${ }^{1,2}$, C. Beck ${ }^{2}$, and V. Martínez Pillet ${ }^{2}$ \\ ${ }^{1}$ University of Colorado, Boulder, CO 80309, USA \\ e-mail: alysa.derks@colorado.edu \\ 2 National Solar Observatory, 3665 Discovery Drive, Boulder, CO 80303, USA \\ e-mail: [cbeck,vmpillet]@nso.edu
}

Received 23 May 2017 / Accepted 24 January 2018

\begin{abstract}
Context. Polarimetric observations taken with ground- or space-based telescopes usually need to be corrected for changes of the polarization state in the optical path.

Aims. We present a technique to determine the polarization properties of a telescope through observations of spectral lines that have no or negligible intrinsic linear polarization signals. For such spectral lines, any observed linear polarization must be induced by the telescope optics. We apply the technique to observations taken with the Spectropolarimeter for Infrared and Optical Regions (SPINOR) at the Dunn Solar Telescope (DST) and demonstrate that we can retrieve the characteristic polarization properties of the DST at three wavelengths of 459,526 , and $615 \mathrm{~nm}$.

Methods. We determine the amount of crosstalk between the intensity Stokes $I$ and the linear and circular polarization states Stokes $Q$, $U$, and $V$, and between Stokes $V$ and Stokes $Q$ and $U$ in spectropolarimetric observations of active regions. We fit a set of parameters that describe the polarization properties of the DST to the observed crosstalk values. We compare our results to parameters that were derived using a conventional telescope calibration unit (TCU).

Results. The values for the ratio of reflectivities $X=r_{s} / r_{p}$ and the retardance $\tau$ of the DST turret mirrors from the analysis of the crosstalk match those derived with the TCU within the error bars. We find a negligible contribution of retardance from the entrance and exit windows of the evacuated part of the DST. Residual crosstalk after applying a correction for the telescope polarization stays at a level of 3-10\% regardless of which parameter set is used, but with an rms fluctuation in the input data of already a few percent. The accuracy in the determination of the telescope properties is thus more limited by the quality of the input data than the method itself. Conclusions. It is possible to derive the parameters that describe the polarization properties of a telescope from observations of spectral lines without intrinsic linear polarization signal. Such spectral lines have a dense coverage (about $50 \mathrm{~nm}$ separation) in the visible part of the spectrum $(400-615 \mathrm{~nm})$, but none were found at longer wavelengths. Using spectral lines without intrinsic linear polarization is a promising tool for the polarimetric calibration of current or future solar telescopes such as the Daniel K. Inouye Solar Telescope (DKIST).
\end{abstract}

Key words. telescopes - techniques: polarimetric - line: profiles

\section{Introduction}

Most existing solar telescopes were designed without clear requirements for their polarimetric performance and calibration. The telescopes currently available for high-spatial-resolution solar physics are calibrated using an analytical model of their time-varying Mueller matrices. The free parameters in these models are fit to data obtained using calibration polarizers at the entrance of the telescopes (Skumanich et al. 1997; Beck et al. 2005; Selbing 2010; Socas-Navarro et al. 2011) or used literature values for the polarization properties of the mirrors (Collados 1999; Schlichenmaier \& Collados 2002).

The calibration strategy of the Advanced Stokes Polarimeter (ASP; Elmore et al. 1992) at the Dunn Solar Telescope (DST; Dunn 1964; Dunn \& Smartt 1991) as described in Skumanich et al. (1997) paved the way for other telescopes to carry out similar polarimetric characterizations. A key aspect of the ASP/DST calibration was to separate the polarization effects of the telescope with a time-dependent Mueller matrix $\mathbf{T}(t)$ from those of the polarimeter with a static Mueller matrix $\mathbf{X}$ by inserting polarization calibration optics between the two. This point of insertion made the polarimeter matrix $\mathbf{X}$ constant in time but made the telescope Mueller matrix $\mathbf{T}$ time-dependent to account for the varying telescope geometry. The calibration optics provided the polarization properties of all of the optical components downstream from the insertion point, i.e., the polarimeter matrix. Upstream from the insertion point, the data obtained with the entrance polarizer constrained the polarization properties described by the matrix $\mathbf{T}(t)$. Polarization calibration optics usually have a small diameter and can be manufactured to high accuracy compared to the large optics used for entrance polarizers. Placing such high-quality calibration optics as high up in the optical path as feasible is thus beneficial.

The French Télescope Héliographique pour l'Etude du Magnétisme et des Instabilités Solaires (THEMIS; López Ariste et al. 2000) and the proposed Large Earth-based Solar Telescope (LEST; Engvold 1991) followed a different strategy for their polarization calibration. They minimized instrumental polarization effects by mounting the polarization analyzer upstream of any oblique reflection. This design effectively transformed these telescopes into dedicated polarimeters. However, placing the analyzer so high up in the optical beam has several drawbacks. 
THEMIS uses a dual-beam scheme which implies the transfer of two linearly polarized beams through various optical elements which leads to different optical aberrations in them. LEST was designed to use only one of the linearly polarized beams with a loss of $50 \%$ of the photons. Without a dual-beam scheme to minimize the spurious seeing-induced polarization signals (Lites 1987), LEST was forced to rely on high-speed detectors of the Zürich Imaging Polarimeter type (ZIMPOL; Stenflo \& Povel 1985). We also note that the presence of entrance windows in both telescopes prevented them from being completely free of instrumental polarization effects.

In light of these circumstances, all modern solar telescopes adopted the strategy of the ASP and incorporated calibration optics as high up in the optical path as possible, thus calibrating the largest number of optical components. One recent example is the German GREGOR telescope (Schmidt et al. 2012) that uses a polarization calibration unit near the secondary focus of the telescope, right behind the primary and secondary mirrors. The calibration unit is located before breaking the revolution symmetry around the optical axis. Thus, the expected polarization effects from the mirrors upstream are small, originating only from the off-axis configuration outside the center of the field of view and diffraction effects. Sanchez Almeida \& Martinez Pillet (1992) demonstrated that these effects are only important for absolute polarimetry at the $10^{-4}$ level. For GREGOR, the matrix downstream of the calibration optics is geometry- and timedependent, whereas the matrix upstream is constant in time, and, in this case, close to the identity matrix.

The Daniel K. Inouye Solar Telescope (DKIST; McMullin et al. 2016) under construction on the island of Maui will follow a similar strategy as GREGOR. A polarization calibration unit is located downstream of the secondary mirror. In the case of DKIST, the first two mirrors M1 and M2 have an off-axis configuration that generates a telescope matrix $\mathbf{T}$ that deviates significantly from the identity matrix, and is constant in time. The angles of incidence on these two mirrors vary between $6^{\circ}$ and $20^{\circ}$. Accurate ray-tracing with a realistic modeling of the mirror coatings demonstrates that $U \leftrightarrow V$ crosstalk terms of the order of 8\% can be expected in T (Harrington \& Sueoka 2016 , 2017). Interestingly, the same calculations show that the diagonal elements are all close to unity to within less than a percent. Like for GREGOR, all temporal dependencies are transferred to the matrix downstream of the calibration optics. The polarization properties of $\mathbf{T}$ are independent of the telescope pointing and change only with the degradation of the coatings with time. Thus, the inference of this matrix is much simpler than in the case of the DST, the Swedish Solar Telescope (SST), or the German Vacuum Tower Telescope (VTT).

The 4-m aperture of DKIST prevents usage of an entrance linear polarizer. A number of strategies have been devised to calibrate the instrumental polarization to the required level of accuracy of $5 \times 10^{-4}$ (see Socas-Navarro et al. 2005; Socas-Navarro 2005a,b; Elmore et al. 2014). One option for the calibration of the DKIST T-matrix is based on spectral lines that have no intrinsic linear polarization through the Zeeman effect: for example, the Fe II line at $614.9 \mathrm{~nm}$ (Lites 1993). A list of lines with this property, which depends on the atomic transitions involved, was compiled by Sanchez Almeida \& Vela Villahoz (1993) and Vela Villahoz et al. (1994). These authors proposed the use of those lines to measure the asymmetries of Stokes $V$ profiles as they are unaffected by instrumental polarization.

In this paper, we revisit their list of lines without linear polarization to study their suitability for polarimetric calibration. We compare the results obtained from observations of lines without linear polarization taken in 2016 at the DST to those from the standard calibration done in 2010 using the telescope entrance linear polarizers to understand the limitations of the approach. The calibration of the $\mathbf{T}(t)$ matrix for the DST is more complex than the calibration of $\mathbf{T}$ for DKIST, thus succeeding with the DST demonstrates the potential interest of this method for DKIST.

Section 2 describes our selection process to identify suited spectral lines. Section 3 gives the theoretical background for the derivation of telescope properties from observations. The observations used are described in Sect. 4. Sections 5 and 6 provide the data analysis and results, respectively. Section 7 contains the discussion, while Sect. 8 gives our conclusions. The appendices provide the complete line list (Appendix A), a few more data examples at other wavelengths (Appendix B) and error estimates of crosstalk measurements (Appendix C).

\section{Synthesis of spectral lines and line selection}

There exist special atomic transitions that produce spectral lines without any, or with negligible, intrinsic linear polarization (LP) induced by the Zeeman effect (Sanchez Almeida \& Vela Villahoz 1993; Vela Villahoz et al. 1994). These lines can be used to uncover polarization properties of a telescope or to test the quality of the polarimetric calibration because most, or all, linear polarization signal is spurious and indicates polarization crosstalk. We analyzed the two tables of spectral lines without or with small LP provided by Vela Villahoz et al. (1994, their Tables 1 and 2) to find the lines that would be best suited for telescope calibration purposes. We restricted the analysis to lines with rest wavelengths in the range $400-1100 \mathrm{~nm}$. We obtained all necessary information, including excitation potential, $\log (\mathrm{gf})$, transition parameters, and the effective Landé coefficient $\left(g_{\mathrm{eff}}\right)$ from the NIST Atomic Spectral Database ${ }^{1}$. We disregarded any lines for which the value of $\log (\mathrm{gf})$ could not be found. This left 31 lines from Table 1 of Vela Villahoz et al. (1994; see Table B.1) and 56 lines from their Table 2 (see Table B.2).

After the suitable lines were chosen, a spectral synthesis was done using the Stokes Inversion based on Response functions code (SIR; Ruiz Cobo \& del Toro Iniesta 1992). For this synthesis, we used a solar model atmosphere with a magnetic field inclination of $45^{\circ}$ and an azimuth of $22.5^{\circ}$, a macro- and micro-turbulent velocity of $1 \mathrm{~km} \mathrm{~s}^{-1}$, and zero Doppler shift. The magnetic field inclination and azimuth were chosen in order to obtain polarization signal in all Stokes parameters $Q, U$, and $V$, whereas the macro- and micro-turbulence were selected so that realistic polarization amplitudes would be obtained. The synthesis was repeated with three different magnetic field strengths: $100 \mathrm{G}$ (quiet Sun), $1500 \mathrm{G}$ (plage), and $2500 \mathrm{G}$ (sunspot) to simulate regions of different magnetic activity on the solar surface.

Using the synthetic profiles, we made a final selection of lines suitable for calibration purposes based on line depth, polarization amplitude, and absence of line blends. Line blends were identified by a comparison to spectra from the Fourier transform spectrometer (FTS; Kurucz et al. 1984) atlas. We extracted the line depth from the $100 \mathrm{G}$ synthesis, and the maximal Stokes $Q$, $U$, and $V$ amplitudes for all lines from the $1500 \mathrm{G}$ synthesis (see Tables B.1 and B.2). We identified a subset of six lines without or with negligible linear polarization (see Table 1) for testing the approach of inferring polarimetric telescope properties.

\footnotetext{
http://physics.nist.gov/PhysRefData/ASD/lines_form.
} html 

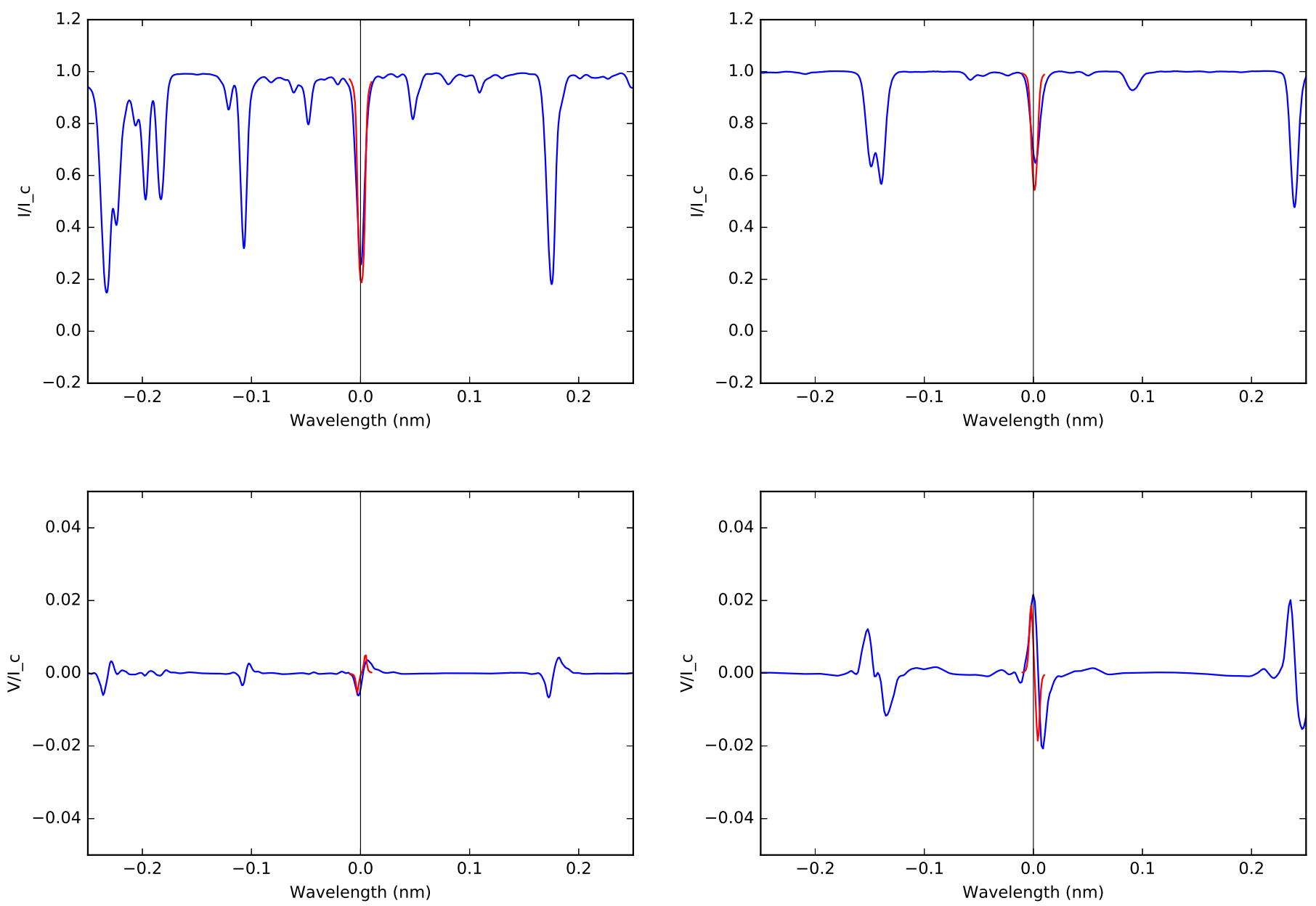

Fig. 1. Synthetic Stokes profiles of Stokes I (top panel) and V (bottom panel) for the $\mathrm{Cr}$ II line at $458.820 \mathrm{~nm}$ in the synthesis with $1500 \mathrm{G}$ shown in red. The blue lines show the corresponding profiles from the FTS atlas.

These lines were selected because of their large line depths and polarization amplitudes, the absence of line blends (cf. Figs. 1-3 and Figs. A.1 and A.2), and a continuous wavelength coverage from 400 to $615 \mathrm{~nm}$. Figures 1-3 show the synthetic profiles of the lines at 458.8, 526.2, and $614.9 \mathrm{~nm}$ analyzed in this study. In these plots, the Stokes $V$ profiles of the FTS atlas were scaled to match the synthesis. Neutral lines, such as Ca I at $526.2 \mathrm{~nm}$, are generally better suited than singly ionized lines because the latter disappear in a cool atmosphere such as that present in sunspots and pores. The Ca I line at $526.2 \mathrm{~nm}$ has small LP (ratio of $V / L P \approx 10$ ) while the lines at 458.8 and $614.9 \mathrm{~nm}$ have zero LP. The only line that has been previously used for calibration purposes is Fe II at $614.9 \mathrm{~nm}$ (Lites 1993; Cabrera Solana et al. 2005; Elmore et al. 2010).

\section{Application of lines without LP for polarimetric calibration}

The following expression is valid for the Stokes vector $S$ after propagation through the telescope optics for a spectral line without LP:

$S=\left(\begin{array}{l}I \\ Q \\ U \\ V\end{array}\right)=\left(\begin{array}{llll}T_{11} & T_{12} & T_{13} & T_{14} \\ T_{21} & T_{22} & T_{23} & T_{24} \\ T_{31} & T_{32} & T_{33} & T_{34} \\ T_{41} & T_{42} & T_{43} & T_{44}\end{array}\right)\left(\begin{array}{c}I^{\prime} \\ 0 \\ 0 \\ V^{\prime}\end{array}\right)$,

Fig. 2. As in Fig. 1 but for Fe II at $614.923 \mathrm{~nm}$.

where $\mathbf{T}$ is the Mueller matrix of the telescope and $\left(I^{\prime}, 0,0, V^{\prime}\right)^{T}$ is the light incident on the telescope. by

The observed linear and circular polarization are then given

$Q=T_{21} \cdot I^{\prime}+T_{24} \cdot V^{\prime}$,

$U=T_{31} \cdot I^{\prime}+T_{34} \cdot V^{\prime}$,

$V=T_{41} \cdot I^{\prime}+T_{44} \cdot V^{\prime}$.

The crosstalk $I \rightarrow Q U V \equiv T_{i 1} \cdot I$ can easily be measured at continuum wavelengths outside of any spectral line and subsequently be compensated for, which yields:

$Q_{\text {corr }}=T_{24} \cdot V^{\prime}$,
$U_{\text {corr }}=T_{34} \cdot V^{\prime}$,
$V_{\text {corr }}=T_{44} \cdot V^{\prime}$.

With that, the ratios $Q_{\text {corr }} / V_{\text {corr }}$ and $U_{\text {corr }} / V_{\text {corr }}$ correspond to the ratios of telescope matrix entries $T_{24} / T_{44}$ and $T_{34} / T_{44}$. With a determination of $Q_{\text {corr }} / V_{\text {corr }}$ and $U_{\text {corr }} / V_{\text {corr }}$ from observational data taken at a given telescope, one can thus obtain the ratio of telescope matrix entries at different times and telescope geometries. If a model of the polarimetric properties of the corresponding telescope is available, one can then infer the set of model parameters that best reproduce the observed $Q_{\text {corr }} / V_{\text {corr }}$ and $U_{\text {corr }} / V_{\text {corr }}$ ratios. The approach can also be used in the opposite direction for test purposes. If a telescope correction 

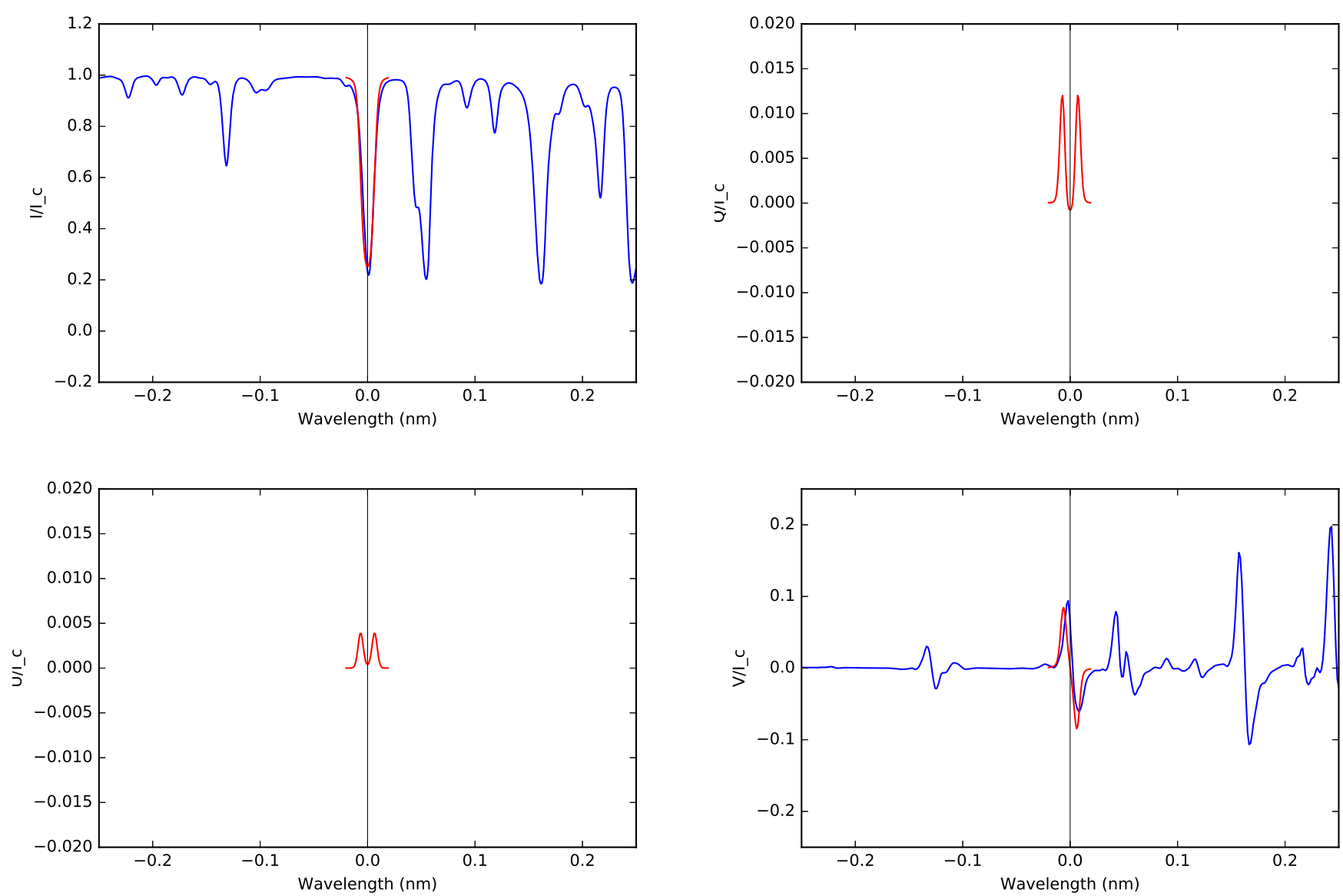

Fig. 3. Synthetic Stokes profiles of Stokes I (top left), $Q$ (top right), $U$ (bottom left), and $V$ (bottom right) for the Ca I line at $526.171 \mathrm{~nm}$ in the synthesis with $1500 \mathrm{G}$. There is no corresponding FTS atlas data with Stokes $Q$ and $U$ measurements.

Table 1. Transition parameters, line depth, and maximal polarization amplitudes at $1500 \mathrm{G}$.

\begin{tabular}{|c|c|c|c|c|c|c|c|c|c|c|c|}
\hline Element & $\begin{array}{c}\text { Ionization } \\
\text { state }\end{array}$ & $\begin{array}{c}\lambda_{0} \\
{[\mathrm{~nm}]}\end{array}$ & $\begin{array}{c}\text { Excitation } \\
\text { potential }[\mathrm{eV}]\end{array}$ & $\log (g f)$ & Transition & $g_{\mathrm{eff}}$ & LP? & $\begin{array}{c}\text { Line } \\
\text { depth [\%] }\end{array}$ & $\begin{array}{l}\text { Max. } \\
|Q / I|\end{array}$ & $\begin{array}{l}\text { Max. } \\
|U / I|\end{array}$ & $\begin{array}{l}\text { Max. } \\
|V / I|\end{array}$ \\
\hline Mn & I & 425.766 & 2.953 & -0.70 & 4D $0.5-4 \mathrm{P} 0.5$ & 1.333 & $\mathrm{No}$ & 81.0 & 0 & 0 & 0.028 \\
\hline $\mathrm{Ti}$ & II & 431.490 & 1.1609 & -1.104 & 4P $0.5-4 \mathrm{D} 0.5$ & 1.333 & No & 92.1 & 0 & 0 & 0.023 \\
\hline $\mathrm{Cr}$ & II & 458.820 & 4.071 & -0.64 & 4D $0.5-6 \mathrm{~F} 0.5$ & 0.333 & No & 81.2 & 0 & 0 & 0.005 \\
\hline $\mathrm{Fe}$ & I & 514.174 & 2.424 & -2.238 & 3P $1.0-3 \mathrm{D} 1.0$ & 1.000 & Low & 84.3 & 0.016 & 0.007 & 0.092 \\
\hline $\mathrm{Ca}$ & I & 526.171 & 2.521 & -0.73 & 3D 1.0-3P 1.0 & 1.000 & Low & 79.7 & 0.012 & 0.004 & 0.085 \\
\hline $\mathrm{Fe}$ & II & 614.923 & 3.889 & -2.8 & 4D $0.5-4 \mathrm{P} 0.5$ & 1.333 & No & 45.6 & 0 & 0 & 0.019 \\
\hline
\end{tabular}

is applied using a set of parameters and there is some residual LP, it can be deduced that the model parameters are incorrect and need to be updated. For lines with small linear polarization, Eqs. (5)-(7) are not strictly valid, but with $V / \mathrm{LP} \approx 10$ the contribution from $T_{22}, T_{23}, T_{32}$, and $T_{33}$ is still very small.

\section{Observations}

To test the usage of spectral lines without linear polarization for the inference of telescope parameters, we ran an observing campaign at the DST on 8-31 March 2016. We combined the Spectropolarimeter for Infrared and Optical Regions (SPINOR; Socas-Navarro et al. 2006), the Facility Infrared Spectropolarimeter (FIRS; Jaeggli et al. 2010), and the Interferometric Bidimensional Spectrometer (IBIS; Cavallini 2006; Reardon \& Cavallini 2008) to obtain spectropolarimetric data in different wavelength regions at the same time. A dichroic beam splitter (BS) was used to separate infrared (IR) and visible (VIS) wavelengths. FIRS was fed with the IR light while the VIS light was split evenly between IBIS and SPINOR by an achromatic 50-50 BS. IBIS and FIRS observed spectral lines with a regular Zeeman pattern $(617.3,630.25,656,854,1083$, and $1565 \mathrm{~nm})$ which were not used for the current study. Both IBIS and FIRS were subsequently dropped from the setup on 17 and 18 March, respectively, and the corresponding BSs in the light feed were removed or replaced with flat mirrors to increase the light level in SPINOR.

SPINOR was set to observe the six lines without or with small LP listed in Table 1 in two different configurations. In the first configuration (setup 1), the lines at 426, 431, and $615 \mathrm{~nm}$ were observed from 8 to 17 March, while the lines at 459, 514, and $526 \mathrm{~nm}$ were covered from 17 to 24 March (setup 2). 


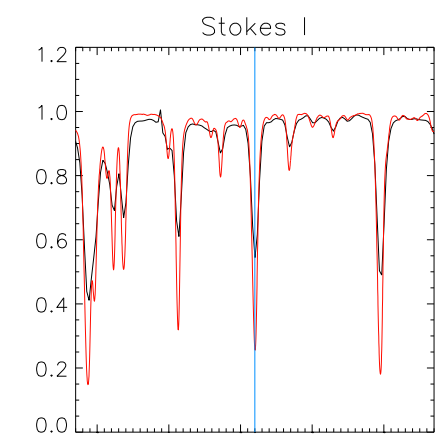

Stokes $U$

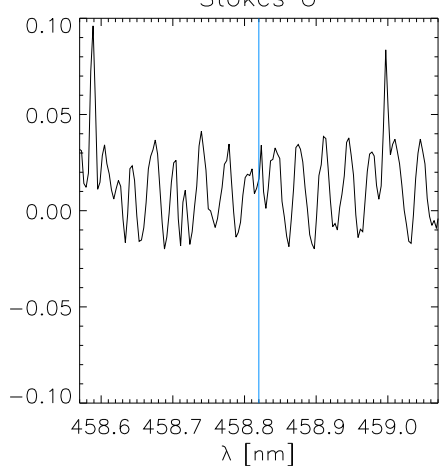

$\lambda[\mathrm{nm}]$

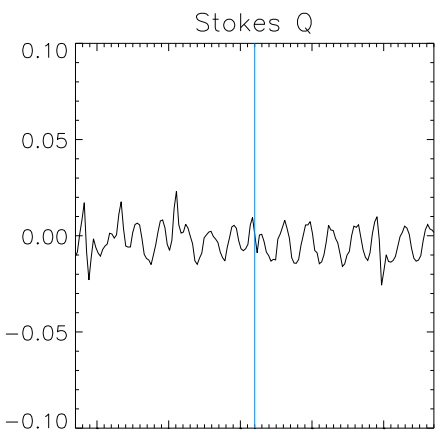

Stokes $V$

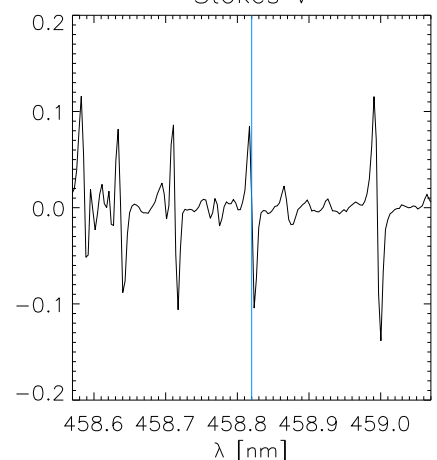

$\lambda[\mathrm{nm}]$

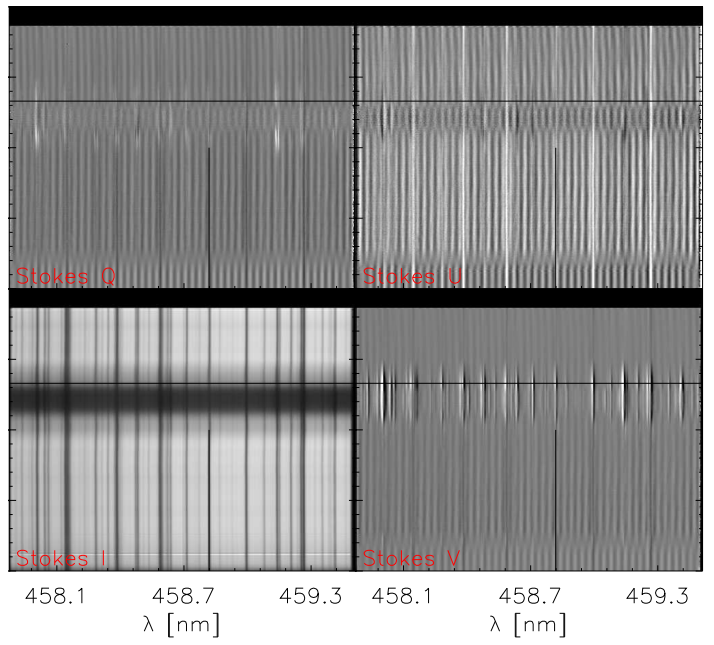

Fig. 4. Observed example spectra at $458.8 \mathrm{~nm}$. Top panels: individual $I Q U V$ profiles from the location indicated with a horizontal black line in the lower panels. The red lines show the corresponding profiles from the FTS atlas. Bottom panels: slit spectra of (clockwise, starting left bottom) IQUV on a cut across the center of a sunspot. The line without LP is indicated by a black vertical bar.

The wavelength range always covered additional lines with a regular Zeeman pattern as well (Figs. 4-6 and Figs. A.3A.5). The spectral sampling was between 3 and $5 \mathrm{pm}$. The exposure time was between 22 and $125 \mathrm{~ms}$ and the integration time was between 10 and $30 \mathrm{~s}$ depending on the setup of SPINOR and the light feed. The spatial sampling in the scanning direction was $0^{\prime \prime} .75\left(0^{\prime \prime} .37\right)$ in setup 1 (setup 2) and the sampling along the slit was about $0^{\prime \prime} .35$ in all cases. The slit width was $100 \mu$ corresponding to about $0^{\prime \prime}$. 75 on the Sun.

In each configuration, several maps of the sunspots in NOAA 12519 and 12524 with $30^{\prime \prime} \times \approx 100^{\prime \prime}$ extent were acquired each day. We also took a few observations of small pores and quiet Sun at disc center to sample regions with smaller polarization
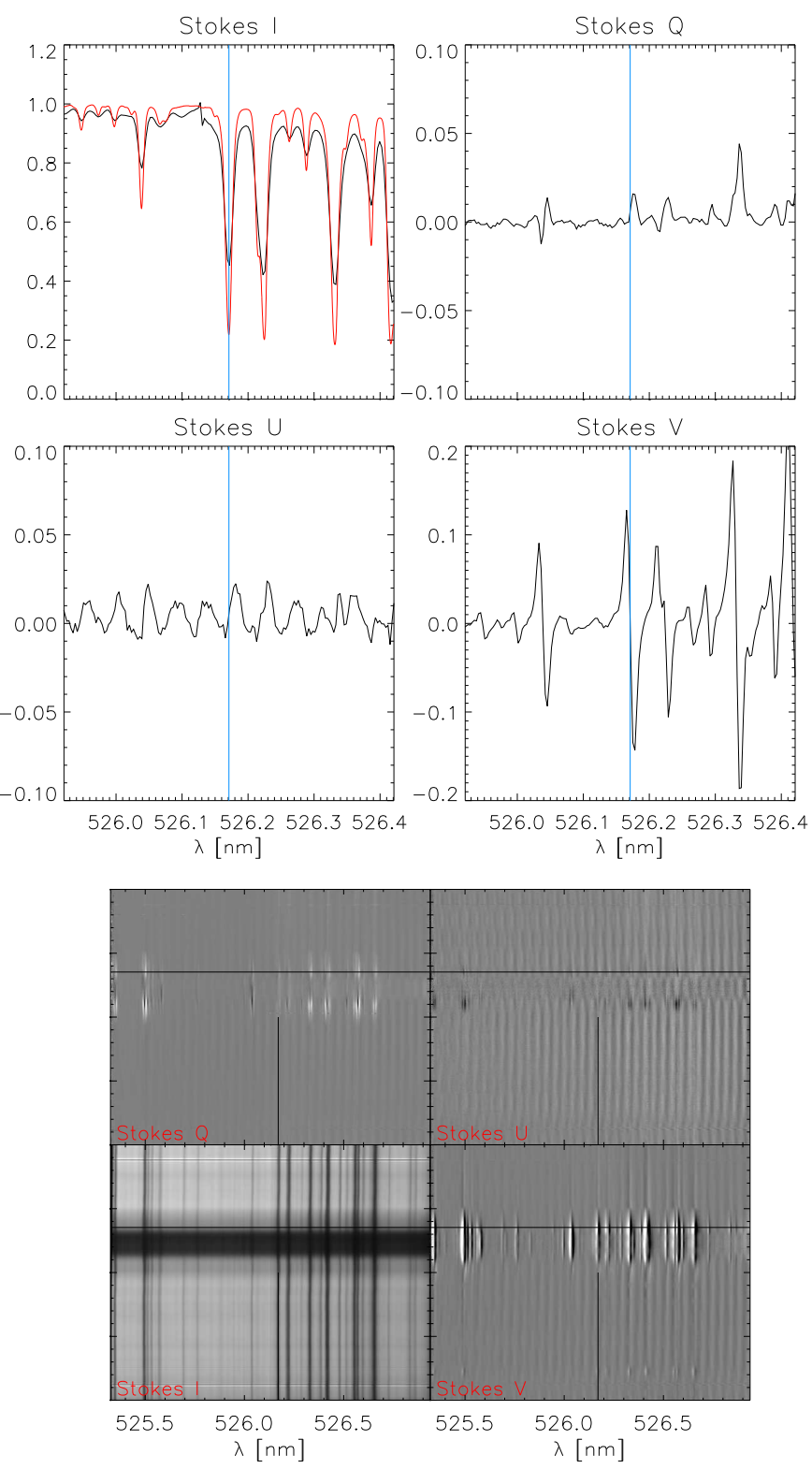

Fig. 5. As in Fig. 4 but for $526.2 \mathrm{~nm}$.

amplitudes for comparison. In total, about 30 datasets were obtained in each setup.

Given the amplitude of the interference fringes at all wavelengths shorter than $450 \mathrm{~nm}$ (Figs. A.3 and A.4) and the fact that 514 and $526 \mathrm{~nm}$ are close to each other, we only analyzed the data at 459,526 , and $615 \mathrm{~nm}$.

\section{Data analysis}

\subsection{Preparation of input data}

This section addresses the methods we used to infer the parameters of the DST telescope model from the observations and the steps required to prepare input data corresponding to Eqs. (5)-(7) for all locations with significant polarization signal.

\subsubsection{Reduction of SPINOR data}

We first reduced the data without applying the correction for the telescope polarization, but with a correction for $I \rightarrow Q U V$ 


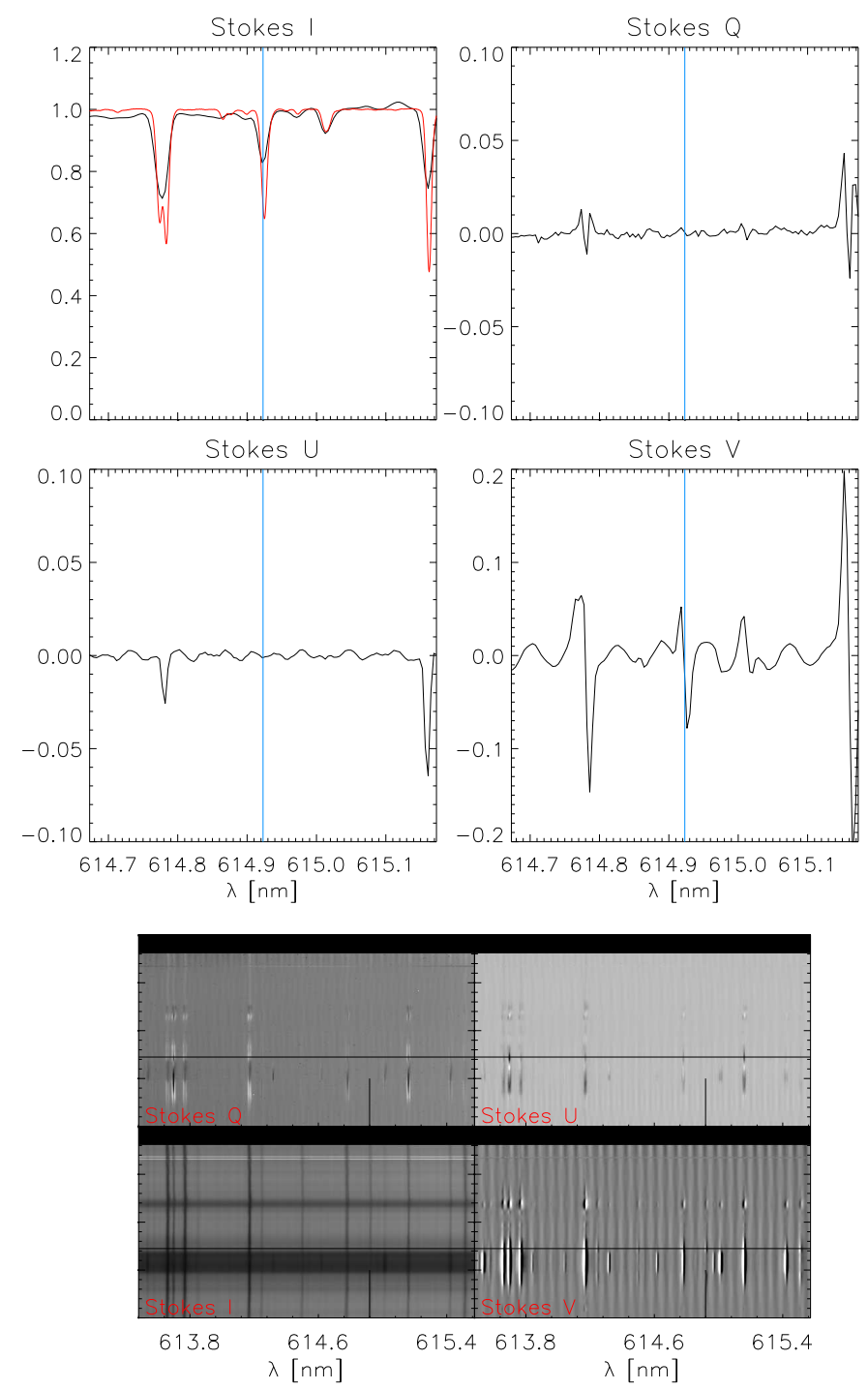

Fig. 6. As in Fig. 4 but for $614.9 \mathrm{~nm}$.

crosstalk, using the SPINOR data pipeline ${ }^{2}$ that was developed in 2013. The $I \rightarrow Q U V$ crosstalk was determined in a continuum wavelength range close to the line of interest without linear polarization. The values of the $I \rightarrow Q U V$ crosstalk were stored for later use because they contain the information on the first column of the telescope matrix. We estimate an error of below $1 \%$ for the $I \rightarrow Q U V$ crosstalk from the presence of the interference fringes (see Appendix C.1). After this step, a twodimensional (2D) Fourier filtering in Stokes $Q U V$ was applied to reduce the fringe amplitude. The Fourier filter was set to remove spectral frequencies within a manually set frequency range that were constant along the slit, i.e., only fringes with a spatial frequency of zero were removed. The fringe pattern in all of the data also contained higher-order fringes that were not taken out. Since the polarization signal can easily extend along 50\% of the slit length in observations of active regions, any attempt to include higher spatial frequencies can remove genuine polarization signal as well. The fringe amplitude, period, and phase were also not constant across the spectrum in both the spatial and spectral domain, thus only the primary fringe component could be corrected for. The example slit spectra in Figs. 4-6 show

\footnotetext{
2 http://nsosp.nso.edu/dst-pipelines
}
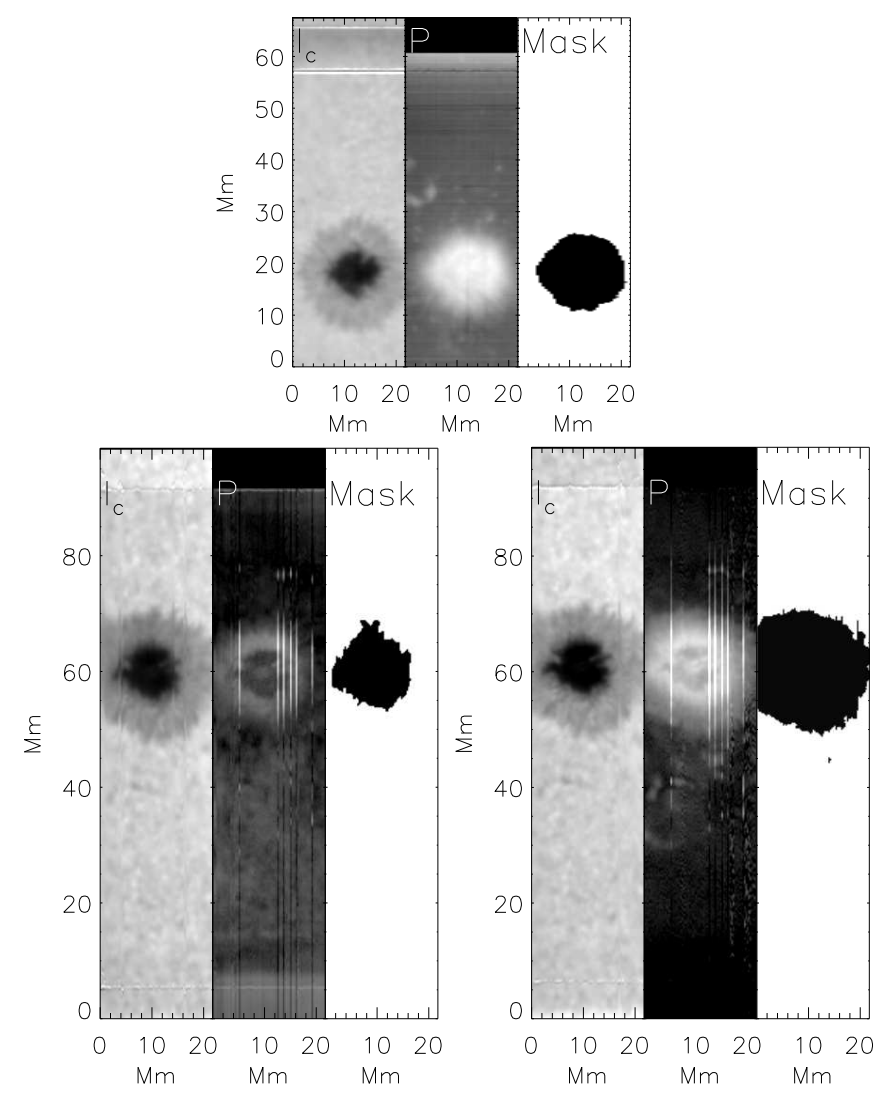

Fig. 7. Maps of one scan at $459 \mathrm{~nm}$ (bottom left panel), $526 \mathrm{~nm}$ (bottom right panel), and $614 \mathrm{~nm}$ (top panel). Left to right: continuum intensity, $I_{c}$, polarization degree $p_{\max }$, and mask of significant polarization signal. The vertical bright stripes in $p_{\max }$ for 459 and $526 \mathrm{~nm}$ were caused by a temporary loss of the synchronization between polarization modulation and exposures during the scanning.

observed spectra after the correction for the strongest fringe pattern. For these example spectra, the telescope correction was applied as well to show the genuine Stokes $Q U V$ signals.

\subsubsection{Determination of locations with significant polarization signal}

To determine the locations inside the FOV that showed significant polarization signal, the maximal polarization degree $p_{\text {max }}$ of every profile was calculated by

$p_{\max }=\max [p(\lambda)]_{\mid \Delta \lambda}=\max \left[\sqrt{Q^{2}+U^{2}+V^{2}} / I(\lambda)\right]_{\mid \Delta \lambda}$

in a small wavelength range $\Delta \lambda$ around a spectral line with strong polarization signal. The line need not has been the one with low or zero LP as each spectral range also covered stronger lines (Figs. 4-6).

From the analysis of all profiles, spatial maps of the polarization degree at 459,526 , and $614 \mathrm{~nm}$ were created for all observations (see Fig. 7). These maps were used to determine the locations of significant polarization signal through a threshold value of $0.01-0.03$ by rejecting all profiles with lower polarization amplitudes. By requiring in addition that the profiles above the threshold belong to a spatially connected area with some minimal size, we masked out everything but sunspots and pores. For the $459 \mathrm{~nm}$ data, in several cases, the fringe pattern in the polarization signal was too strong to use the maps of $p_{\max }$. Instead, a threshold in the map of the continuum intensity was 


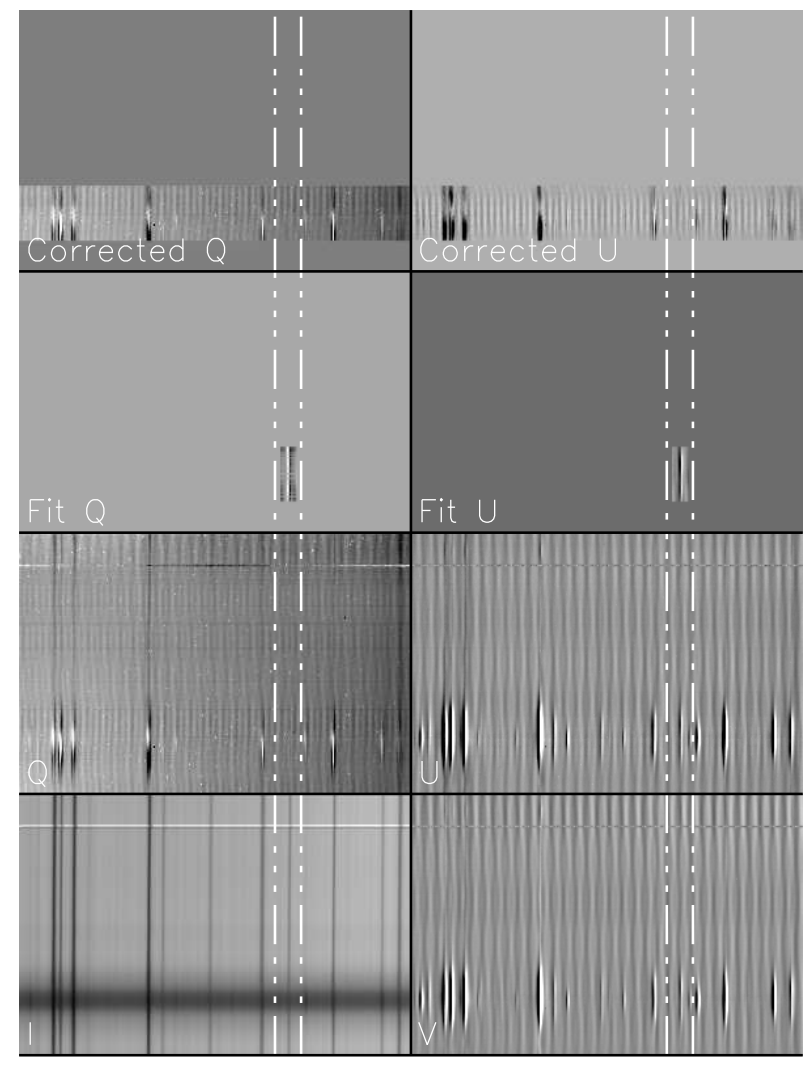

Fig. 8. Example slit spectrum at $615 \mathrm{~nm}$ with the fit of $V \rightarrow Q U$. Left column, bottom to top: Stokes $I$, Stokes $Q$, the fitted $Q=\alpha_{Q} \cdot V$ and the Stokes $Q$ signal after correction, $Q^{\prime}=Q-\alpha_{Q} \cdot V$. Right column, bottom to top: Stokes $V$, Stokes $U$, the fitted $U=\alpha_{U} \cdot V$ and the Stokes $U$ signal after correction, $U^{\prime}=U-\alpha_{U} \cdot V$. The vertical dash-dotted lines enclose the $614.9 \mathrm{~nm}$ line.

used to separate the umbra and penumbra of the sunspot from the brighter quiet Sun and to only retain the profiles located inside the sunspot.

\subsubsection{Determination of $V \rightarrow Q U$ crosstalk}

Determination of the $V \rightarrow Q U$ crosstalk from the ratio of observed profiles $Q / V$ and $U / V$ can become unreliable for small values of $V$ in the presence of noise. Therefore, a linear fit of $Q U_{\text {obs }}(\lambda)=\alpha_{Q U} V_{\text {obs }}$ was made for all profiles above the polarization threshold (see, e.g., Fig. 8). Only the close surroundings of the spectral line of interest without LP were used to avoid contamination with line blends.

To verify the quality of the fit, the obtained crosstalk values $\alpha_{Q U}$ were used to correct the observed $Q$ and $U$ spectra by $Q U^{\prime}=Q U-\alpha_{Q U} \cdot V$ (top row of Fig. 8). The correction can be applied to the full spectral range. In the example of Fig. 8, the change between Stokes $U$ and $U^{\prime}$ is obvious, where the latter has no residual LP in the $614.9 \mathrm{~nm}$ line, while all other Zeeman-sensitive lines in the wavelength range have changed from a Stokes $V$-like shape to regular LP signals by the correction. Thus, the fit to first order correctly retrieves the $V \rightarrow Q U$ crosstalk values. From the final result, we estimate, however, that the error in $V \rightarrow Q U$ caused by the residual fringe pattern is still of the order of a few percent (see Appendix C.2).

The values of $\alpha_{Q U}$ obtained from the individual profiles were averaged along the slit over all profiles in the mask to retrieve $<\alpha_{Q U}>(t)$ because all profiles along the slit were taken at the
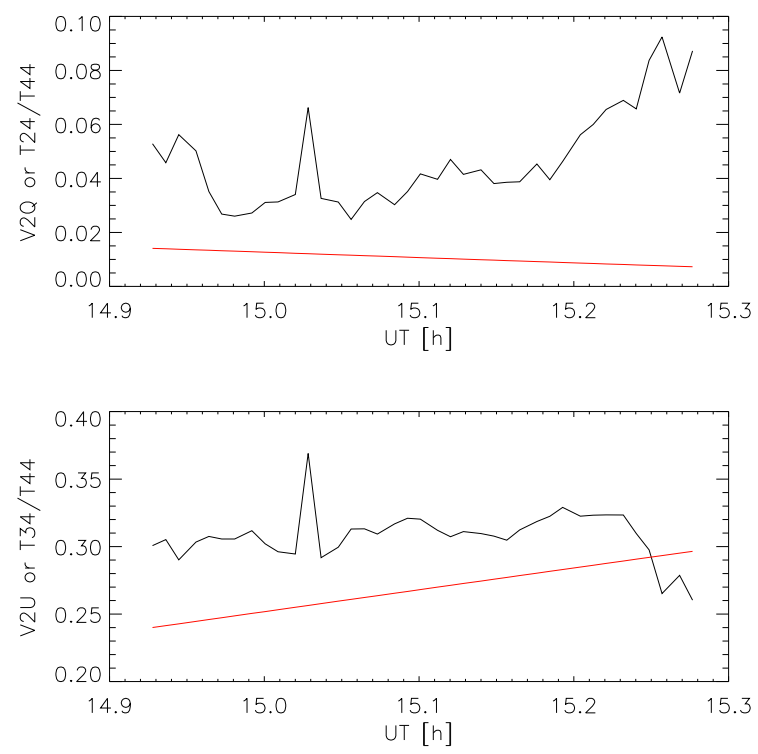

Fig. 9. Crosstalk from $V \rightarrow Q U$ averaged along the slit for the map at $614.9 \mathrm{~nm}$ shown in Fig. 7 (black lines). The red lines show the values of the DST telescope model for $\frac{T_{24}}{T_{44}}$ and $\frac{T_{34}}{T_{44}}$, respectively, using the telescope parameters determined in 2010 .

same time. The time, $t$, is used as a placeholder for the telescope geometry that contains the telescope pointing in elevation and azimuth and the position of the coudé table (table angle). The numbers used in the calculation of the DST telescope model are actually azimuth $(t)$, elevation $(t)$, and table angle $(t)$. Figure 9 shows the values of $\left\langle\alpha_{Q U}>(t)\right.$ at $614.9 \mathrm{~nm}$ for one of the observations. For comparison, the values of the corresponding telescope matrix entries predicted by the "standard" DST telescope model are overplotted. The set of telescope parameters used was derived from measurements acquired in 2010 which were evaluated using the SCAPA code (see Socas-Navarro et al. 2011). The modulus of the values in Fig. 9 agrees to first order, but the agreement is not very close (cf. Sect. 5.2.2 below).

\subsection{Fit of DST telescope model}

\subsubsection{DST telescope model}

The polarization model of the DST has been described in detail in Skumanich et al. (1997) and Socas-Navarro et al. (2011). The optical train consists of the entrance window to the evacuated steel tube, the two flat turret mirrors at an angle of incidence (AOI) of $45^{\circ}$ in an alt-azimuth mounting, the primary mirror at an AOI of $<1^{\circ}$ and the exit window of the vacuum tube. Both turret mirrors are described with the same set of parameters, the ratio of reflectivities parallel and perpendicular to the plane of reflection, $X=r_{\mathrm{s}} / r_{\mathrm{p}}$, and the retardance $\tau_{\text {mirror }}$. The primary mirror is modeled as an ideal mirror because of its small AOI. Entrance (EN) and exit (EX) windows are modeled independently as ideal retarders with two parameters each: the retardance $\tau_{\mathrm{EN}, \mathrm{EX}}$ and the orientation of the fast axis $\beta_{\mathrm{EN}, \mathrm{EX}}$. To capture the exact orientation of the telescope model relative to the instrument calibration unit that is located on the rotating coudé table, a constant offset angle $\theta_{\text {offset }}$ between the telescope model and the coudé table is used as a free parameter. The known geometry of the telescope at any given moment in time is expressed by the elevation, azimuth, and the relative orientation of the coudé table. 

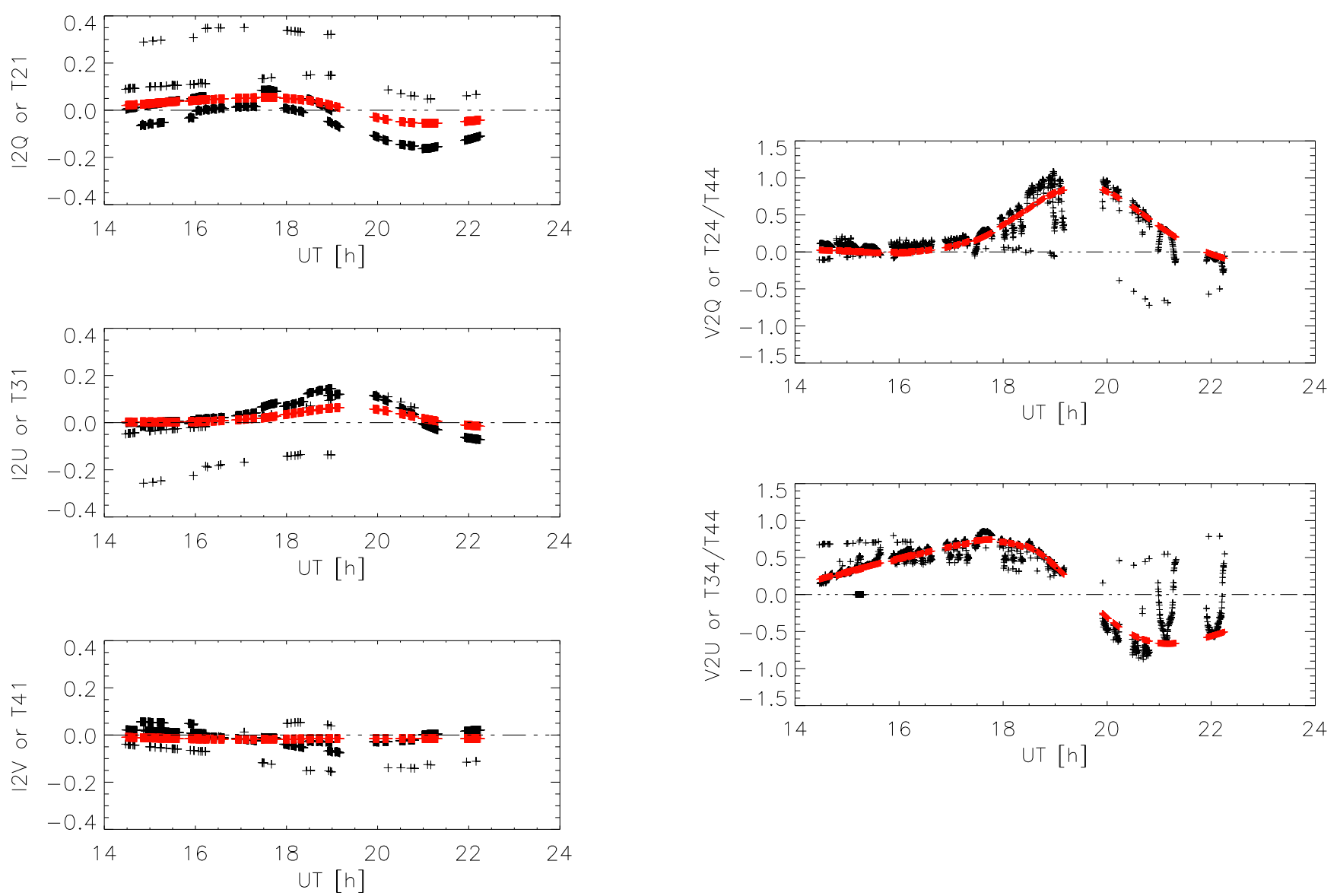

Fig. 10. Input to the fit of telescope parameters derived from all observations of $526.2 \mathrm{~nm}$ during the campaign. Left column, top to bottom: observed $I \rightarrow Q U V$ crosstalk (black crosses). Right column, top to bottom: $V \rightarrow Q U$ crosstalk. The red crosses show the corresponding values of the DST telescope model using the 2010 model parameters.

The free parameters of the DST telescope model are usually determined from measurements with a telescope calibration unit (TCU). The TCU consists of an array of sheet polarizers that is placed on top of the entrance window. The TCU is motorized and can be rotated in a full circle. The evaluation of such data and the results are described in detail in Socas-Navarro et al. (2011). In the current study, we used the parameter set that was determined using the TCU in 2010 as a reference.

\subsubsection{Input data for fitting the telescope model}

The input data for the fit of the telescope model parameters have two components (see, i.e., Fig. 10), the values of the $I \rightarrow Q U V$ crosstalk that correspond to the first column of the telescope matrix, and the values of the $V \rightarrow Q U$ crosstalk that correspond to the ratios of the matrix entries $T_{24} / T_{44}$ and $T_{34} / T_{44}$. Both quantities were determined in individual profiles and then averaged along the slit because each slit spectrum corresponds to one moment in time. The geometrical input parameters (elevation, azimuth, table angle) were extracted from the file headers of each spectrum. In the following figures, we plot all the values solely as a function of time for simplicity. The data for each wavelength range were taken within about a week, so the solar position was similar at the same time on different days. All calculations, however, always used the actual telescope geometry at the moment of the observations.

After plotting the corresponding curves for the data at any of the three wavelengths, we noticed a parabolic pattern in the
$V \rightarrow Q U$ crosstalk data which did not match the 2010 telescope model, but rather touched its predicted values only at the central scan step (see Fig. 10). The parabolic shape is very obvious for some of the individual maps (see the left half of Fig. 11). It presumably was caused by the specific setup for the observations. The SPINOR modulator had been removed from its location in the collimated beam upstream of all instruments. It had been placed as close to the slit as possible to prevent interference with the polarization measurements of FIRS and IBIS and to minimize the image motion caused by a wedge in the modulator optics. The spatial scanning of SPINOR is achieved by moving part of the spectrograph, i.e., the slit unit, the first fold mirror, and the collimator, laterally. The spatial scanning therefore moved the slit relative to the modulator, sampling different areas on the modulator depending on the scan position. The polarimetric calibration of the instrument, however, was only done with the slit centered. Together, this presumably led to a variation of the polarization modulation across the FOV that was not removed by the calibration process. We thus decided to drop all of the data points apart from the central slit position of each map.

We repeated a similar observing run in April 2017 only using SPINOR. In that case, the SPINOR modulator could be left in the collimated beam far upstream of the instrument. The right half of Fig. 11 shows the corresponding values of the $V \rightarrow Q$ and $V \rightarrow U$ crosstalk for one map observed in 2017 at $614.9 \mathrm{~nm}$. The parabolic shape is absent, like for all other data taken in 2017, and all of the scan steps with significant polarization signal 

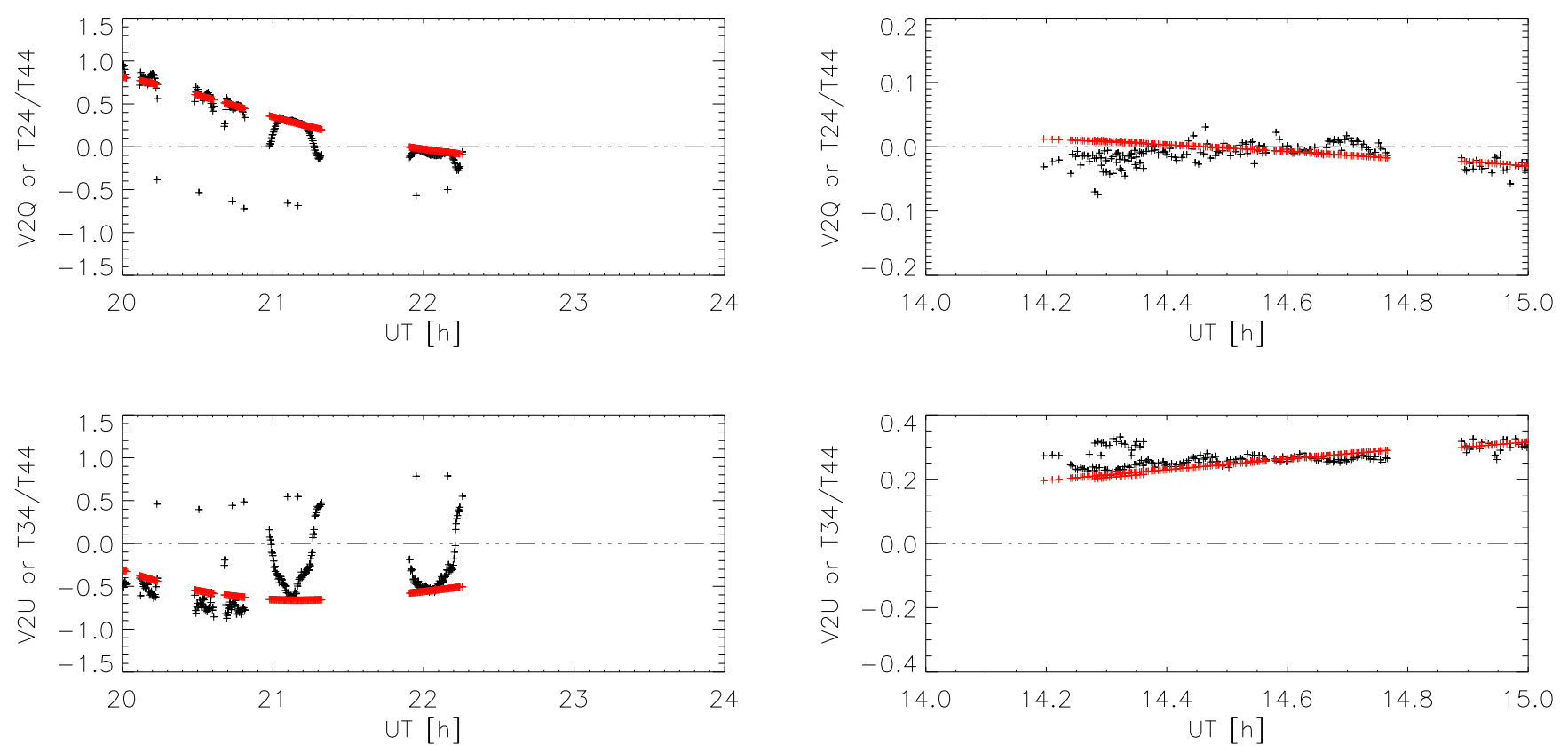

Fig. 11. Left column: magnification of the $V \rightarrow Q U$ crosstalk at $526.2 \mathrm{~nm}$ for observations taken between UT 20 and 24 . Individual maps lasted for approximately $30 \mathrm{~min}$. Most scans show a dominant parabolic shape of the crosstalk values (seen also in Fig. 10). Only the central position of the scan yields a value that agrees with the 2010 telescope model. Right column: same for data taken in 2017 at 614.9 nm with the SPINOR modulator in a collimated beam.

in this map can be used. This confirms that the behavior in the 2016 data was caused by the mechanical spatial scanning across a spatially resolved modulator close to the slit plane.

\subsubsection{Fit of telescope model parameters}

We then attempted to fit the open parameters of the DST telescope model, $X$ and $\tau$ for the turret mirrors, and the retardance $\tau_{\text {EN,EX }}$ and the orientation of the fast axis $\beta_{\text {EN,EX }}$ of the entrance and exit windows to the observed $I \rightarrow Q U V$ and $V \rightarrow Q U$ crosstalk values considering only the central step of each observation.

It was immediately obvious that there is a sign conflict between the observations and the model in the $I \rightarrow Q U V$ crosstalk (orange and red or blue plus symbols in Fig. 12) that were opposite to one another. A mismatch of signs between the 2010 model values determined with SCAPA and the more recent 2013 SPINOR data pipeline was already known. For the application of the telescope correction using the 2010 values in the 2013 pipeline, the offset angle $\theta_{\text {off }}$ of about $90^{\circ}$ in 2010 had to be changed to $\theta_{\text {off }}+90 \sim 180^{\circ}$. There are unfortunately multiple reasons that could cause this sign conflict. A difference in the selection of beams when merging the Stokes vectors would flip the signs of Stokes $Q, U$, and $V$. The linear polarizer of the calibration unit (CU) was taken out of and installed back in the CU a few times between 2010 and 2016. If the optical axis got switched by $90^{\circ}$ when replacing the polarizer, that also would flip the signs in some of the Stokes parameters.

Given the number of possible options, we were not able to uniquely identify the source for the sign mismatch. We therefore used an ad-hoc correction by flipping the sign of the observed $I \rightarrow Q U V$ crosstalk values and used an offset angle of $180^{\circ}$ as initial value. The $V \rightarrow Q U$ values are not directly affected because they represent a ratio of Stokes parameters. For the evaluation of the data in the current study the sign flip thus has no effect. In the evaluation of solar scientific observations, the sign flip would show up as a change of the azimuth of the magnetic field in the line-of-sight reference frame by $90^{\circ}$.

A second point that became obvious instantly was that the retardances of the windows were not constrained by the data. When trying to fit the retardances of the windows and of the turret mirrors at the same time, the former was running off to unreasonably large values of more than $20^{\circ}$ in retardance. Depending on the initial values of the fit, the retardances of the two windows were sometimes changing in opposite directions with approximately a zero net retardance (see also Socas-Navarro et al. 2011, their Sect. 3).

We therefore modified the fit to a two-step procedure. In the first step, only $X, \tau$, and $\theta_{\text {off }}$ were allowed to vary, while in the second step the retardance and position angle of the entrance window and $\theta_{\text {off }}$ were varied. The exit window was not considered and set to a unity matrix. The retardance of the mirrors was found to already be able to reproduce the observations very well, so that the window's retardance in the second step stayed close to zero. The final fit procedure in our case then only used $X, \tau, \theta_{\text {off }}, \beta_{\mathrm{EN}}$, and $\tau_{\mathrm{EN}}$.

\section{Results}

\subsection{Fit quality}

Figure 12 shows the final result of the fit (blue pluses) together with the corresponding curves when using the 2010 model parameters (red pluses) and the observations (black pluses) for all three wavelengths. The input data at $458.8 \mathrm{~nm}$ show the largest scatter that presumably results from the residual fringe pattern (Fig. 4). The differences between the new determination and the 2010 values are rather small, only a few percent. Both parameter sets provide a good fit to the input data. The match of observations and telescope model with any parameter set is slightly worse in the afternoon for times later than about UT 19:00. 

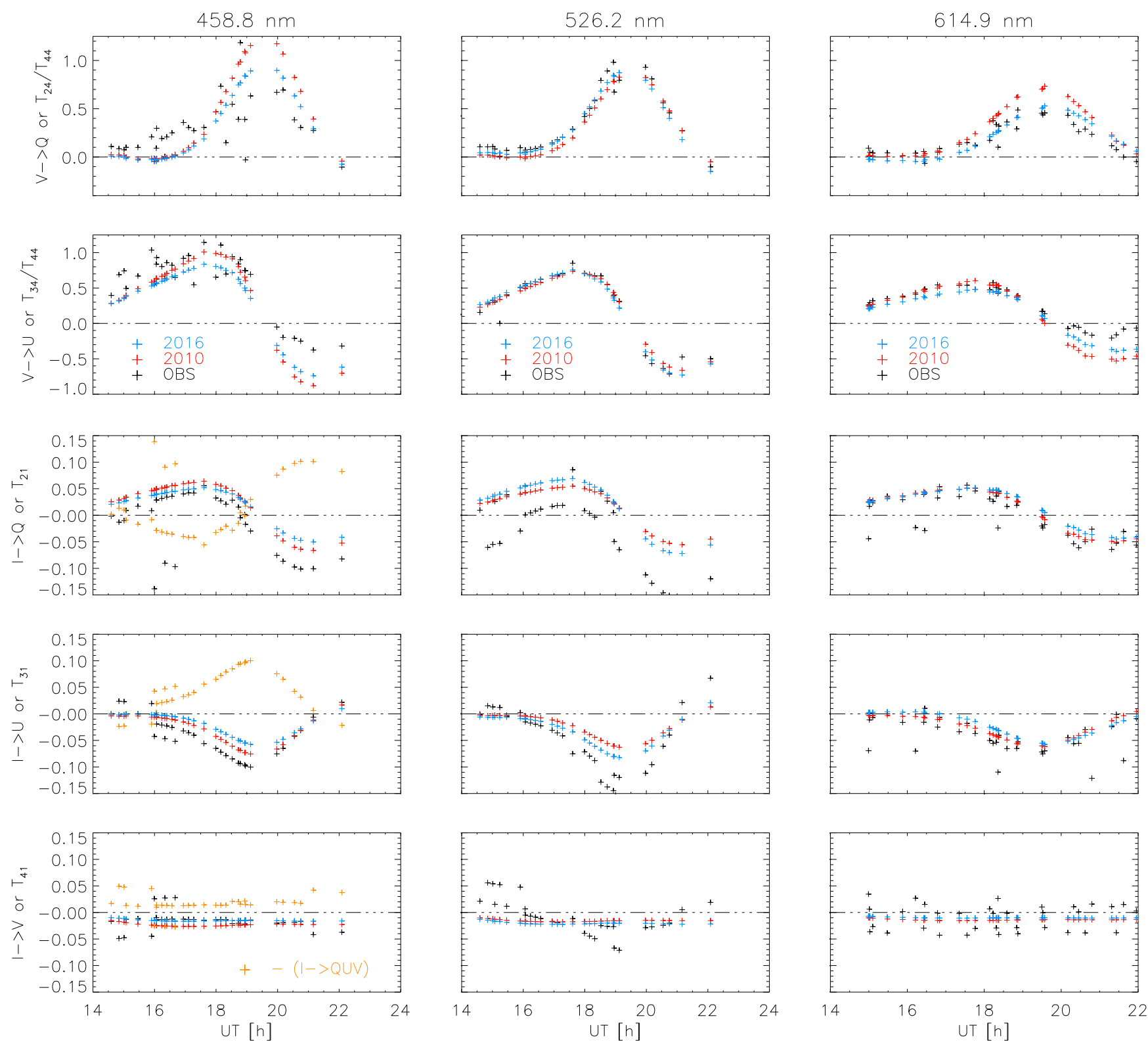

Fig. 12. Fit of telescope parameters at $458.8,526.2$, and $614.9 \mathrm{~nm}$. Top to bottom: observed crosstalk of $V \rightarrow Q$ (black crosses), $V \rightarrow U, I \rightarrow Q$, $I \rightarrow U$, and $I \rightarrow V$. The blue (red) crosses show the corresponding values of the DST model using the 2016 (2010) model parameters. The orange pluses in the lower three panels of the leftmost column show the original $I \rightarrow Q U V$ values before flipping their sign.

\subsection{Ratio of reflectivities $X=r_{\mathrm{s}} / r_{\mathrm{p}}$}

Table 2 lists the results for all free fit parameters from the current determination and the 2010 measurements. In addition, we calculated the values of $X$ and $\tau$ for the turret mirrors assuming optically thick coatings (i.e., no influence of the underlying glass substrate), using the literature values at each wavelength given in the Handbook of Chemistry and Physics (Lide 1994). For the ratio of reflectivities $X$, there is no clear wavelength trend in either the 2010 or 2016 results, while the literature values show a monotonic increase with increasing wavelength. All values for $X$ determined from measurements at the DST are around $1.09 \pm 0.1$, while the literature values are about $0.02-0.05$ lower (see also Socas-Navarro et al. 2011, their Fig. 8). The value of $X$ is only weakly constrained by the $V \rightarrow Q U$ crosstalk. It cancels out for a single mirror (see, i.e., Beck et al. 2005) because, for instance,
$V \rightarrow Q \equiv T_{24} / T_{44}=\frac{-2 X \sin \tau}{2 X \cos \tau}=\tan \tau \neq \mathrm{f}(X)$.

At the DST, there is a slight dependence of $V \rightarrow Q$ on $\mathrm{X}$ because the two turret mirrors rotate relative to each other. The sensitivity test described in Sect. 6.4 below confirms this. Within our assumed error range, the measurements of $X$ from all data acquired at the DST agree well.

\subsection{Window and mirror retardance $\tau$}

The mirror retardance alone was found to already cover the needed values and to provide a good fit to the amplitude of the daily variation in $V \rightarrow Q U$. The second step of the two-step fit using the entrance window retardance as described in Sect. 5.2.3 above yielded a window retardance $\tau_{\mathrm{EN}}$ of $<1^{\circ}$, therefore making the window seem negligible. We note that the initial value of 
Table 2. Literature values, best-fit results, and error estimates of telescope parameters.

\begin{tabular}{cccccc}
\hline \hline & $X$ & $\tau$ & $\theta_{\text {off }}$ & $\tau_{\mathrm{EN}}$ & $\beta_{\mathrm{EN}}$ \\
\hline \multicolumn{5}{c}{$458.8 \mathrm{~nm}$} \\
\hline Literature & 1.057 & 165.8 & - & - & - \\
2010 TCU & 1.101 & 148.9 & 93.1 & 3.0 & 138.6 \\
2016 fit & 1.077 & 153.2 & 178.2 & 0.6 & 22.0 \\
$\sigma$ & \pm 0.033 & \pm 0.5 & \pm 0.7 & \pm 0.6 & - \\
\hline \multicolumn{5}{c}{$526.2 \mathrm{~nm}$} \\
\hline Literature & 1.059 & 167.7 & - & - & - \\
2010 TCU & 1.083 & 155.0 & 93.1 & 2.3 & 138.6 \\
2016 fit & 1.108 & 154.3 & 182.0 & 0.6 & 22.0 \\
$\sigma$ & \pm 0.035 & \pm 0.5 & \pm 0.7 & \pm 0.6 & - \\
\hline \multicolumn{7}{c}{$614.9 \mathrm{~nm}$} \\
\hline Literature & 1.065 & 169.5 & - & - & - \\
2010 TCU & 1.080 & 157.8 & 93.1 & 2.1 & 138.6 \\
2016 fit & 1.074 & 162.7 & 174.2 & 0.4 & 22.0 \\
$\sigma$ & \pm 0.036 & \pm 0.6 & \pm 1.1 & \pm 0.8 & - \\
$\chi^{2} \pm 10 \%$ & \pm 0.150 & \pm 2.6 & \pm 4.0 & \pm 4.0 & - \\
\hline
\end{tabular}
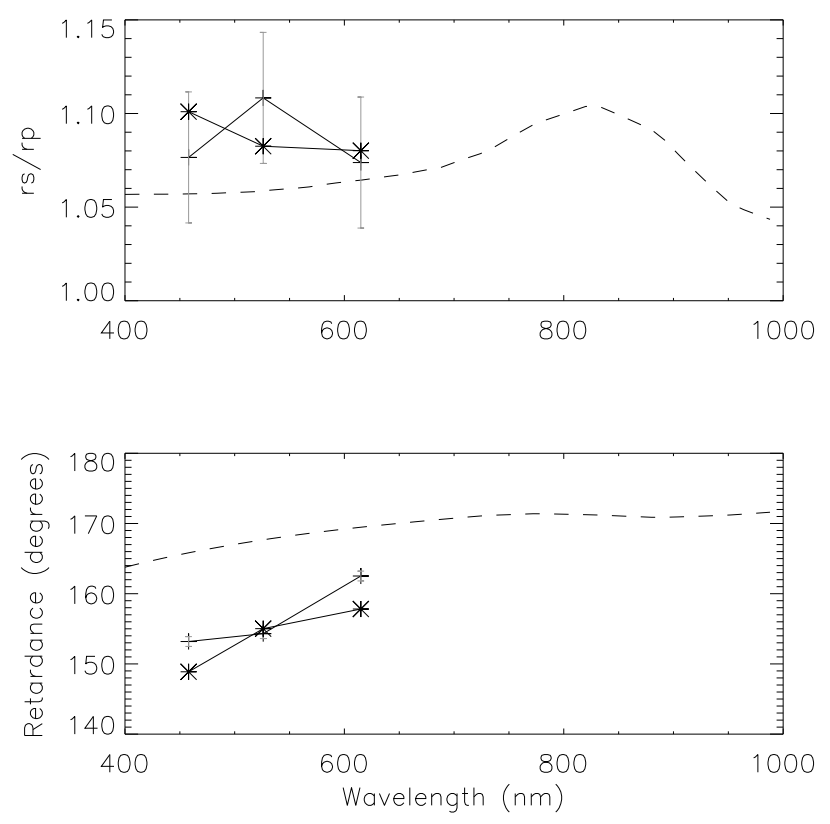

Fig. 13. Values of $X$ (top panel) and $\tau$ (bottom panel) for the literature values (dashed lines), the 2010 telescope model parameters (asterisks), and the current 2016 fit results (crosses). The vertical gray lines show error estimates of $\pm 1^{\circ}$ (bottom panel) and \pm 0.035 (top panel).

the orientation of the optical axis of the window was $22^{\circ}$. This was not significantly changed in the fit (last column of Table 2), which would support the assumption that the window does not contribute to the polarization properties of the optical train, or its contribution cannot be separated from that of the mirrors given the input data.

The retardance of the turret mirrors $\tau$ increases monotonically with wavelength in all cases, that is, for the 2010 fit, the 2016 fit, and the literature values. The 2010 and 2016 fit have values that differ by about $5^{\circ}$, while the literature values are offset from them by more than $10^{\circ}$. The literature values do not take any information obtained at the DST into account, so their global offset from both fit values is uncritical, whereas the fit values themselves agree to first order.

Figure 13 shows a plot of both $X$ and $\tau$ for the turret mirrors for all available values for completeness. The error bars were taken from the estimates in the following section.

\subsection{Sensitivity test and error estimate}

To check the sensitivity of the fit and the telescope model to changes in the model parameters, we varied $X$ and $\tau$ of the turret mirrors by -0.1 to 0.1 and -10 to 10 around the best-fit value, while keeping the window properties at the best-fit values. Figure 14 shows the reaction of the telescope model in the $I \rightarrow Q U V$ and $V \rightarrow Q U$ crosstalk values to these changes at a wavelength of $614.9 \mathrm{~nm}$. The other two wavelengths at 459 and $526 \mathrm{~nm}$ showed a similar behavior.

The $V \rightarrow Q U$ crosstalk reacts only weakly to changes in $X$. Only for values of $X$ that were far outside the range of variation used here, i.e., $X<0.5$ or $X>2$, was a significant change seen. This is due to the fact that the two turret mirrors rotate relative to each other when tracking the Sun, while $X$ cancels out for a single mirror as shown by Eq. (9) above. The $I \rightarrow Q U V$ crosstalk, on the other hand, reacts strongly to changes in $X$, while it is nearly unaffected by changes in $\tau$.

We used the sensitivity test to estimate a reliability interval for the fit procedure by determining the variation of $X$ or $\tau$ that led to a change of the $I \rightarrow Q U V$ or $V \rightarrow Q U$ crosstalk by 0.01 on average. The scatter in the observational values, i.e., for $I \rightarrow Q U V$ (see Appendix C), is of about that order of magnitude, and a variation of $1 \%$ on average caused a clearly visible displacement between the observations and the model output. For instance, for determining the error of $X$, we calculated the average difference between the observed and telescope model values by

$D=\frac{1}{3 N} \sum_{i=1 \ldots N} \sum_{j=2 \ldots 4}\left|T_{j 1}^{\mathrm{obs}}-T_{j 1}(X, \tau)\right|_{i}$,

where $N$ is the number of data points and $T_{j 1}^{\mathrm{obs}}=I \rightarrow Q, U, V$ for $j=2,3,4$.

We then modified the best-fit solution $X_{\text {best-fit }}$ by $\pm \Delta X$ until a value of $D=0.01$ was reached. For the error of $\tau$, we used $V \rightarrow Q U$ in the calculation instead. This yielded a range of about \pm 0.035 for $X, \pm(0.5-0.8)$ degrees for the retardances $\tau$ of the turret mirrors and the entrance window and $\pm 1^{\circ}$ for the offset angle (see Table 2). At the very small retardance best-fit value of the entrance window of less than a degree, the orientation of the window's fast axis was nearly unconstrained. Neither $\chi^{2}$ nor the curves from the telescope model over the day showed a significant response to changes in $\beta_{\mathrm{EN}}$. We therefore could not derive any reasonable error estimate for this parameter. All three spectral ranges gave very similar results. As a second estimate for the error, we varied the parameters around the best-fit solution at $614.9 \mathrm{~nm}$ until $\chi^{2}$ changed by $\pm 10 \%$. If the $\chi^{2}$-surface near the best-fit solution can be approximated by an $n$-dimensional Gaussian, where $n$ is the number of free parameters, then a change of $\chi^{2}$ by $10 \%$ corresponds to a width of the Gaussian of about one $\sigma$. This yielded errors that were larger by a factor of about 4, which seems to be somewhat of an overestimate of the true error.

The offset angle varies by up to $8^{\circ}$ between the different wavelengths even if it should reflect rather a mechanical, 

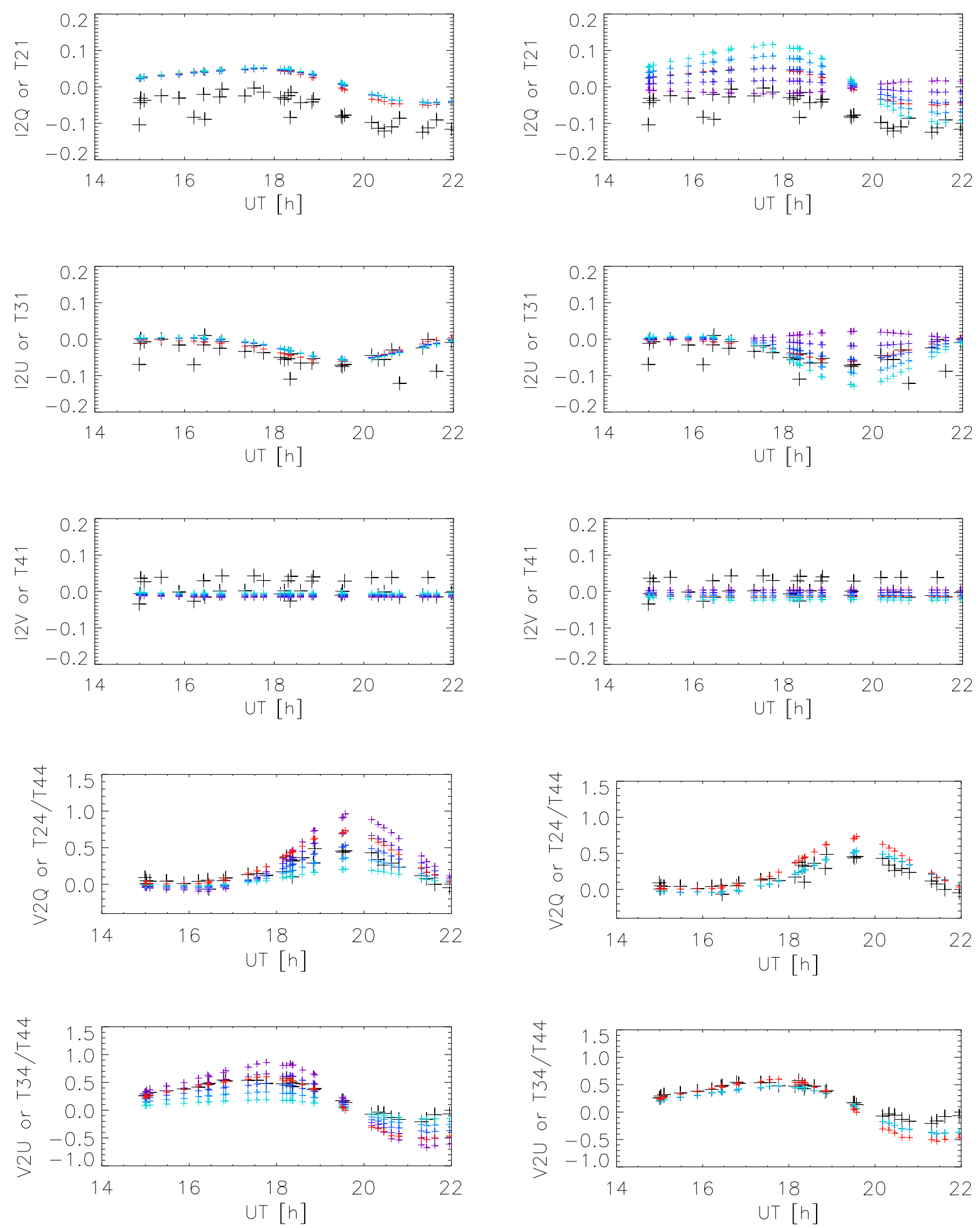

Fig. 14. Sensitivity test of the telescope model at a wavelength of $614.9 \mathrm{~nm}$. Top three rows: change in $I \rightarrow Q U V$ for a variation of the mirror retardance $\tau$ (left column) and the ratio of reflectivities $X$ (right column). Observations are given by black plus symbols, while the red plus symbols correspond to the 2010 parameter set. $\tau$ and $X$ were varied from -10 to 10 and -0.1 to 0.1 , respectively. The resulting curves are indicated by the series of blue to purple plus symbols. Bottom two rows: change in $V \rightarrow Q U$ for a variation of the mirror retardance $\tau$ (left column) and the ratio of reflectivities $X$ (right column).

constant property than any wavelength-dependent optical property. To test the impact of this large variation on the other parameters, we re-ran the fit at $614.9 \mathrm{~nm}$ for the maximal, minimal, and average value of $\theta_{\text {off }}$ while keeping the offset angle fixed. Table 3 shows that the value of $\theta_{\text {off }}$ has an impact on the final $\chi^{2}$ of the fit, but only leads to a minor variation in the mirror parameters $X$ and $\tau$ that is within their estimated error range. The offset angle represents a physical rotation and changes the phase of the daily variation and the relative fraction of Stokes $Q$ and $U$, whereas the main constraint for the mirror parameters is the amplitude of the daily variation (Fig. 14). 

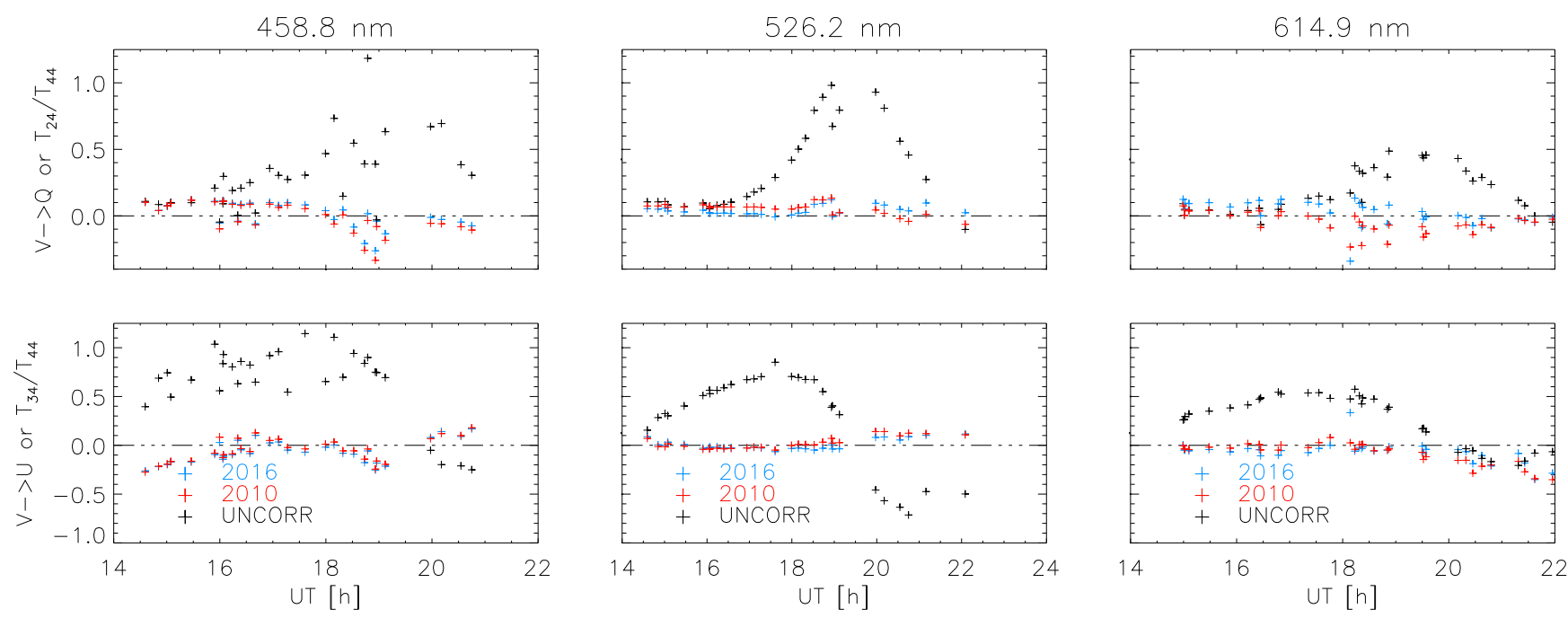

Fig. 15. Residual $V \rightarrow Q$ (top row) and $V \rightarrow U$ (bottom row) crosstalk after applying the telescope correction. Left to right: at 458.8, 526.2, and $614.9 \mathrm{~nm}$. Black plus symbols: observations without telescope correction. Red/blue plus symbols: observations with a telescope correction using the 2010/2016 model parameters.

Table 3. Fit parameters at $614.9 \mathrm{~nm}$ for three different fixed values of the offset angle.

\begin{tabular}{cccc}
\hline \hline$\chi^{2}$ & $X$ & $\tau$ & $\theta_{\text {off }}$ \\
\hline 6.167 & 1.078 & 162.4 & 182.0 \\
5.192 & 1.075 & 162.0 & 178.1 \\
4.808 & 1.071 & 162.7 & 174.4 \\
\hline
\end{tabular}

\subsection{Residual crosstalk with telescope correction}

To test the fit quality, we applied the telescope correction to the observations using the parameter set from 2010 and 2016 prior to running the determination of the $I \rightarrow Q U V$ and $V \rightarrow Q U$ crosstalk. With the telescope correction, all crosstalk values should be close to zero. Figure 15 shows that this is indeed the case for the $V \rightarrow Q U$ crosstalk. The rms fluctuations of the $V \rightarrow Q U$ crosstalk are given in Table 4 for all wavelengths. With the application of the telescope correction, they reduce from the uncorrected case with about $20-30 \%$ rms to $3-10 \%$ rms after the correction. The amplitude of the residual crosstalk in fully calibrated data after the application of the telescope correction matches the fit residuals (see Appendix C.2) which correspond to the mismatch between observations and model prior to the application of the inverse telescope matrix. The new determination in 2016 yields a slightly lower rms than the 2010 parameter set, but usually only by about $1 \%$. The residual crosstalk level thus remains at a value of $3-10 \times 10^{-2}$ regardless of which correction is applied.

\section{Summary and discussion}

We acquired a series of spectropolarimetric observations of active regions containing sunspots over a few days in six different spectral lines without or with negligible linear polarization signals (cf. Table 1). From the analysis of the crosstalk between intensity and polarization, $I \rightarrow Q U V$, and the crosstalk between circular and linear polarization, $V \rightarrow Q$ and $V \rightarrow U$, we were able to infer the parameters that characterize the model of the polarization properties of the DST for a subset of three spectral
Table 4. Residual rms $V \rightarrow Q U$ crosstalk.

\begin{tabular}{lccc}
\hline \hline & $V \rightarrow Q$ & $V \rightarrow U$ \\
\hline & \multicolumn{3}{c}{$458.8 \mathrm{~nm}$} \\
\hline Uncorrected & 0.27 & 0.43 \\
2010 TCU & 0.11 & 0.12 \\
2016 fit & 0.10 & 0.12 \\
\hline & \multicolumn{3}{c}{$526.2 \mathrm{~nm}$} \\
\hline Uncorrected & 0.33 & 0.47 \\
2010 TCU & 0.04 & 0.06 \\
2016 fit & 0.03 & 0.05 \\
\hline & & $614.9 \mathrm{~nm}$ \\
\hline Uncorrected & 0.17 & & 0.25 \\
2010 TCU & 0.08 & 0.11 \\
2016 fit & 0.09 & 0.11 \\
\hline
\end{tabular}

lines, and hence three wavelength regions at 459, 526, and $615 \mathrm{~nm}$ (cf. Table 2).

\subsection{Method}

Unlike most other calibration techniques in use for current ground-based solar telescopes (Skumanich et al. 1997; Beck et al. 2005; Selbing 2010; Socas-Navarro et al. 2011), this approach does not require any additional optics other than a spectropolarimeter with medium to high spectral resolution. The method does not rely on any assumptions for lines without intrinsic linear polarization, while for the other lines the linear polarization is assumed to be negligibly small (Lites 1993; Sanchez Almeida \& Vela Villahoz 1993; Vela Villahoz et al. 1994; Li et al. 2017).

The analysis procedure is automatic to a high degree. After identification of the corresponding spectral line to be used, the thresholds in polarization degree or continuum intensity for the selection of spatial positions to be included and the initial values for the least-squares fit are the only items which must be manually provided.

The method is based on a statistical approach. The data to be analyzed do not have to be acquired consecutively over a few 
days as done in our case, but can be collected over a longer period of time as long as the telescope properties do not vary significantly within that time frame. In addition, the spatial resolution of the observations does not need to be very high because the generation of crosstalk between polarization states only requires a significant signal in the source polarization state. This offers the possibility to build up a base dataset with sufficient statistics over a time frame of weeks to months during periods of bad seeing.

In the same way that high spatial resolution is not ultimately required, there is also no requirement for observing active regions, pores, or sunspots. Polarization amplitudes of a few percent in Stokes $V$ are also reached by magnetic network to elements (e.g., Rezaei et al. 2007). An accurate determination of the crosstalk between polarization states then only requires a high signal-to-noise ratio to detect signals of a fraction of a percent, i.e., signals of $10^{-4}-10^{-3}$ amplitude must be above the noise floor.

Several of the spectral lines without or with negligible linear polarization signal that can be used for the application of the method (see Tables B.1 and B.2) result from neutral atoms, unlike the singly ionized line at $614.9 \mathrm{~nm}$. The advantage of lines pertaining to neutral elements is that their line strength does not weaken in "cold" solar structures such as pores and sunspots, while singly ionized lines can disappear completely exactly where the magnetic field strength and polarization signal is largest (see Fig. 6).

Although there is a dense wavelength coverage from the blue end of the visible spectrum at $400 \mathrm{~nm}$ up to $615 \mathrm{~nm}$, there were no suitable lines found in the red end of the spectrum $(>615 \mathrm{~nm})$.

\subsection{Performance}

As for most methods used to determine polarization properties, it is difficult to provide a good estimate of the accuracy of the approach. We find a residual $V \rightarrow Q U$ crosstalk of about $5-10 \%$ after application of the correction for the telescope polarization based on our 2016 best-fit values (Table 4). The residual crosstalk is comparable to that when using the 2010 telescope parameter set that was derived with a TCU. However, as seen in Figs. 12 and 15 , there is already a significant scatter of a few percent in the input data that is presumably caused by residual interference fringes, and is thus due to the quality of the input data, not the approach itself.

The values of the telescope parameters of our current determination match those of the 2010 telescope model within the error bars, although both are offset from literature values (Fig. 13). The match of the values determined from actual measurements at the DST is a positive sign, as the applicability of the literature values assuming thick coatings to the DST mirrors is not ensured. Therefore, based on our findings, we propose that this new calibration method can be just as accurate as the standard DST calibration with a TCU depending on the data quality.

The main limitation for the application of the approach to the current data is the interference fringes in the observations; they impact the determination of crosstalk values between different Stokes parameters at a level of several percent.

\section{Conclusions and future work}

We conclude that it is possible to derive the parameters that describe the polarization properties of a telescope from observations of spectral lines without or with negligible linear polarization signal. These spectral lines cover much of the blue side of the visible part of the spectrum, but no suitable lines were found above $615 \mathrm{~nm}$.

It is impossible to use a conventional TCU consisting of linear polarizers for the 4-m DKIST telescope. Using spectral lines without intrinsic linear polarization is thus a promising approach for its polarization calibration. At the DST, a timedependent system with a variable geometric configuration has to be calibrated. The corresponding problem for DKIST is much less complex as only the first two mirrors need to be characterized which are static with fixed angles of incidence and a fixed relative orientation.

We would also like to search for suitable lines towards the red end of the spectrum and develop a similar method for lines with a regular Zeeman pattern, for which one would need to make assumptions either about the magnetic field geometry or about the symmetry properties of Zeeman signals.

Acknowledgements. The Dunn Solar Telescope in Sunspot/NM is operated by the National Solar Observatory (NSO). The NSO is operated by the Association of Universities for Research in Astronomy, Inc. (AURA) under cooperative agreement with the National Science Foundation.

\section{References}

Beck, C., Schlichenmaier, R., Collados, M., Bellot Rubio, L., \& Kentischer, T. 2005, A\&A, 443, 1047

Cabrera Solana, D., Bellot Rubio, L. R., \& del Toro Iniesta, J. C. 2005, A\&A, 439, 687

Cavallini, F. 2006, Sol. Phys., 236, 415

Collados, M. 1999, in Third Advances in Solar Physics Euroconference: Magnetic Fields and Oscillations, eds. B. Schmieder, A. Hofmann, \& J. Staude, Astronomical Society of the Pacific Conference Series, 184, 3

Dunn, R. B. 1964, Appl. Opt., 3, 1353

Dunn, R. B., \& Smartt, R. N. 1991, Adv. Space Res., 11, 139

Elmore, D. F., Lites, B. W., Tomczyk, S., . et al. 1992, in Polarization Analysis and Measurement, eds. D. H. Goldstein, \& R. A. Chipman, Proc. SPIE, 1746 22

Elmore, D. F., Lin, H., Socas Navarro, H., \& Jaeggli, S. A. 2010, in GroundBased and Airborne Instrumentation for Astronomy III, Proc. SPIE, 7735, $77354 \mathrm{E}$

Elmore, D. F., Sueoka, S. R., \& Casini, R. 2014, in Ground-Based and Airborne Instrumentation for Astronomy V, Proc. SPIE, 9147, 91470F

Engvold, O. 1991, Adv. Space Res., 11, 157

Harrington, D. M., \& Sueoka, S. R. 2016, in Advances in Optical and Mechanical Technologies for Telescopes and Instrumentation II, Proc. SPIE, 9912, 99126U

Harrington, D. M., \& Sueoka, S. R. 2017, J. Astron. Telesc. Instrum. Syst., 3, 018002

Jaeggli, S. A., Lin, H., Mickey, D. L., et al. 2010, Mem. Soc. Astron. Italiana, 81, 763

Kurucz, R. L., Furenlid, I., Brault, J., \& Testerman, L. 1984, Solar Flux Atlas from 296 to $1300 \mathrm{~nm}$, National Solar Obs. (New Mexico: Sunspot)

Li, W., Casini, R., del Pino Alemán, T., \& Judge, P. G. 2017, ApJ, 848, 82

Lide, D. R. 1994, CRC Handbook of Chemistry and Physics, 75th ed. (Boca Raton, FL: CRC Press)

Lites, B. W. 1987, Appl. Opt., 26, 3838

Lites, B. W. 1993, Sol. Phys., 143, 229

López Ariste, A., Rayrole, J., \& Semel, M. 2000, A\&AS, 142, 137

McMullin, J. P., Rimmele, T. R., Warner, M., et al. 2016, in Ground-Based and Airborne Telescopes VI, Proc. SPIE, 9906, 99061B

Reardon, K. P., \& Cavallini, F. 2008, A\&A, 481, 897

Rezaei, R., Schlichenmaier, R., Beck, C. A. R., Bruls, J. H. M. J., \& Schmidt, W. 2007, A\&A, 466, 1131

Ruiz Cobo, B., \& del Toro Iniesta, J. C. 1992, ApJ, 398, 375

Sanchez Almeida, J., \& Martinez Pillet, V. 1992, A\&A, 260, 543

Sanchez Almeida, J., \& Vela Villahoz E. 1993, A\&A, 280, 688

Schlichenmaier, R., \& Collados, M. 2002, A\&A, 381, 668

Schmidt, W., von der Lühe, O., Volkmer, R., et al. 2012, Astron. Nachr., 333, 796 
A. Derks et al.: Inferring telescope polarization through lines without linear polarization

Selbing, J. 2010, MSc Thesis, ArXiv e-prints [arXiv: 1010.4142]

Skumanich, A., Lites, B. W., Pillet, V. M., \& Seagraves, P. 1997, ApJS, 110, 357

Socas-Navarro, H. 2005a, J. Opt. Soc. Am. A, 22, 539

Socas-Navarro, H. 2005b, J. Opt. Soc. Am. A, 22, 907

Socas-Navarro, H., Elmore, D. F., Keller, C. U., et al. 2005, in Solar Physics and Space Weather Instrumentation, eds. S. Fineschi, \& R. A. Viereck, Proc. SPIE, 5901, 52
Socas-Navarro, H., Elmore, D., Pietarila, A., et al. 2006, Sol. Phys., 235, 55

Socas-Navarro, H., Elmore, D., Asensio Ramos, A., \& Harrington, D. M. 2011, A\&A, 531, A2

Stenflo, J. O., \& Povel, H. 1985, Appl. Opt., 24, 3893

Vela Villahoz, E., Sanchez Almeida, J., \& Wittmann, A. D. 1994, A\&AS, 103, 293 


\section{Appendix A: Line spectra at 426, 431, and $514 \mathrm{~nm}$}

Figures A.1 and A.2 show the profiles of the lines of Mn I at $425.766 \mathrm{~nm}$ (no LP), Ti II at $431.490 \mathrm{~nm}$ (no LP), and Fe I at $514.174 \mathrm{~nm}$ (small LP) from the synthesis with a magnetic field strength of $1500 \mathrm{G}$.

Corresponding example spectra from the observations are shown in Figs. A.3-A.5. The Ti II line at $431.5 \mathrm{~nm}$ is partly blended with other Zeeman-sensitive lines. The residual fringe amplitude of still several percent after removal of the strongest
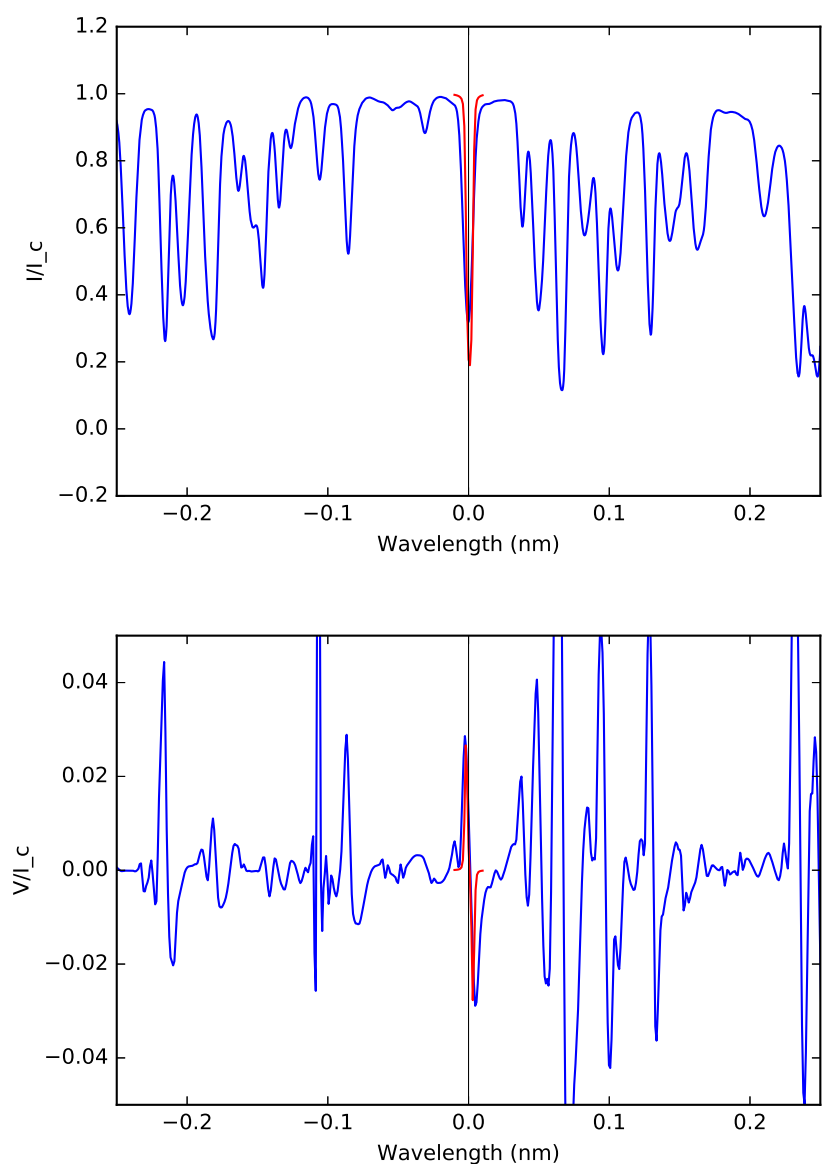

interference fringes makes any evaluation of the data at 425.8 and $431.5 \mathrm{~nm}$ unreliable. The observed peak Stokes $V$ polarization amplitudes of both lines of about 0.1 are sufficient for the inference of telescope properties as demonstrated with the $\mathrm{Ca}$ I line at $526.2 \mathrm{~nm}$ that has a maximal Stokes $V$ amplitude of 0.085 . The Fe I line at $514.2 \mathrm{~nm}$ is well isolated in the spectrum (Figs. A.2 and A.5) and does not suffer strongly from interference fringes in the observations, but its wavelength range was already covered by the Ca I line at $526 \mathrm{~nm}$, so we did not evaluate these data.
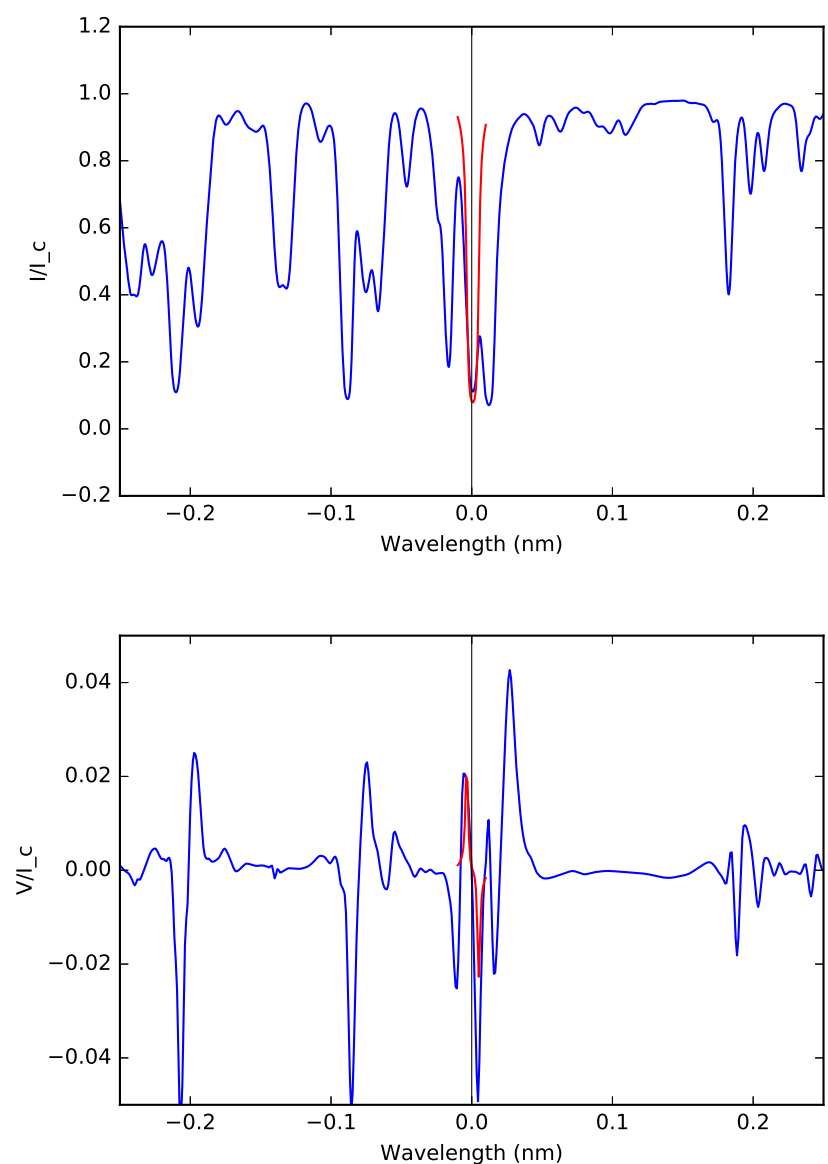

Fig. A.1. Synthetic Stokes profiles of Stokes I (top panel) and $V$ (bottom panel) for the Mn I line at $425.766 \mathrm{~nm}$ (left column) and Ti II at 431 . $490 \mathrm{~nm}$ in the synthesis with $1500 \mathrm{G}$. The blue lines show the corresponding profiles from the FTS atlas. 
A. Derks et al.: Inferring telescope polarization through lines without linear polarization
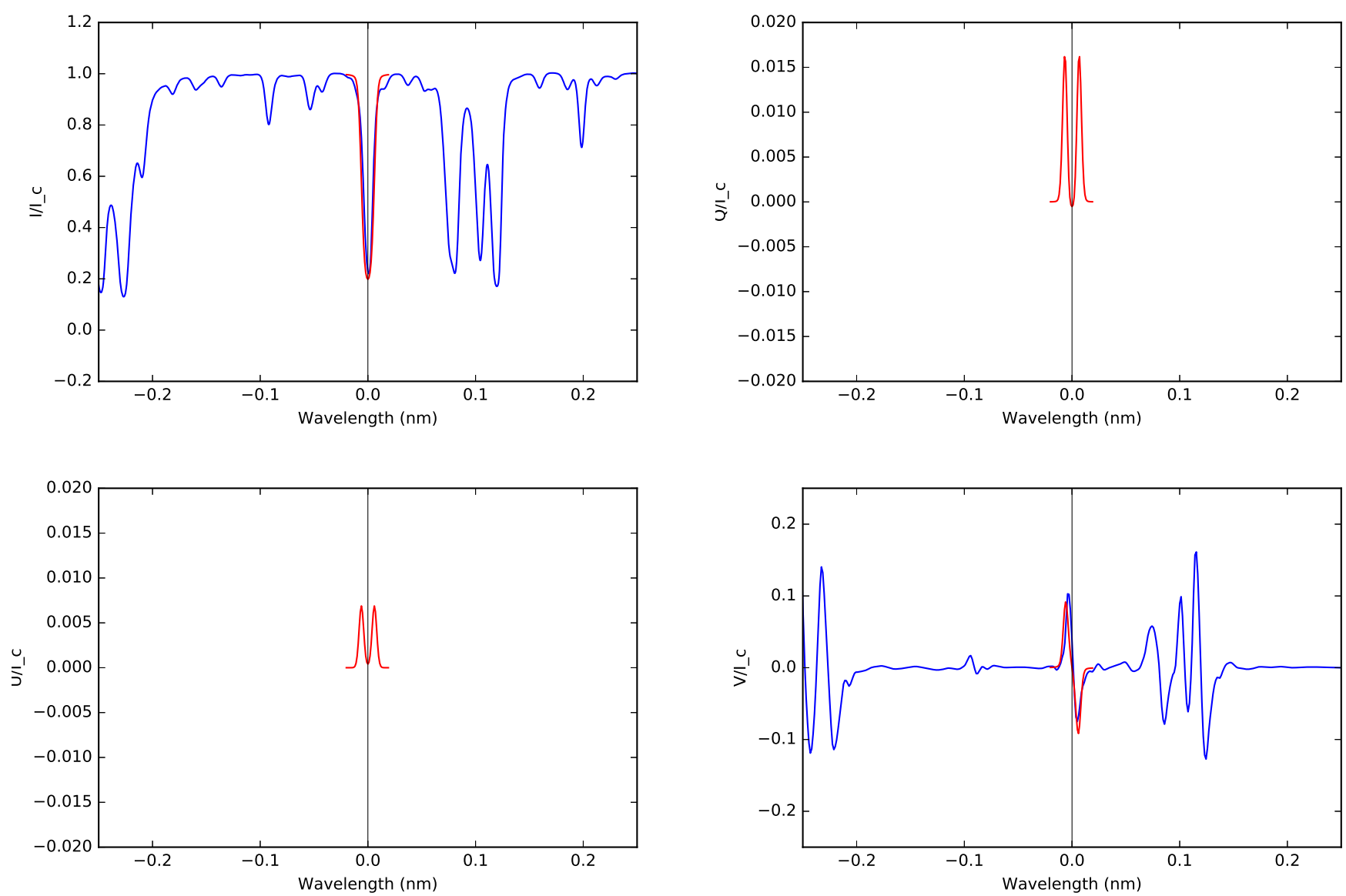

Fig. A.2. Synthetic Stokes profiles of Stokes I (top left), $Q$ (top right), $U$ (bottom left), and $V$ (bottom right) for the Fe I line at $514.174 \mathrm{~nm}$ in the synthesis with $1500 \mathrm{G}$.

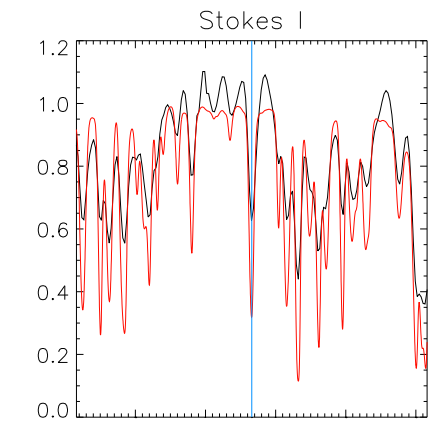

Stokes U
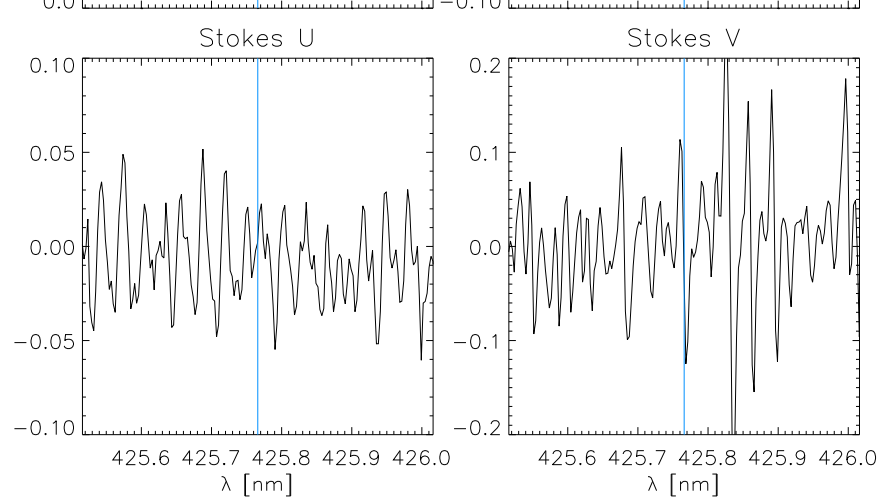

$\lambda[\mathrm{nm}]$
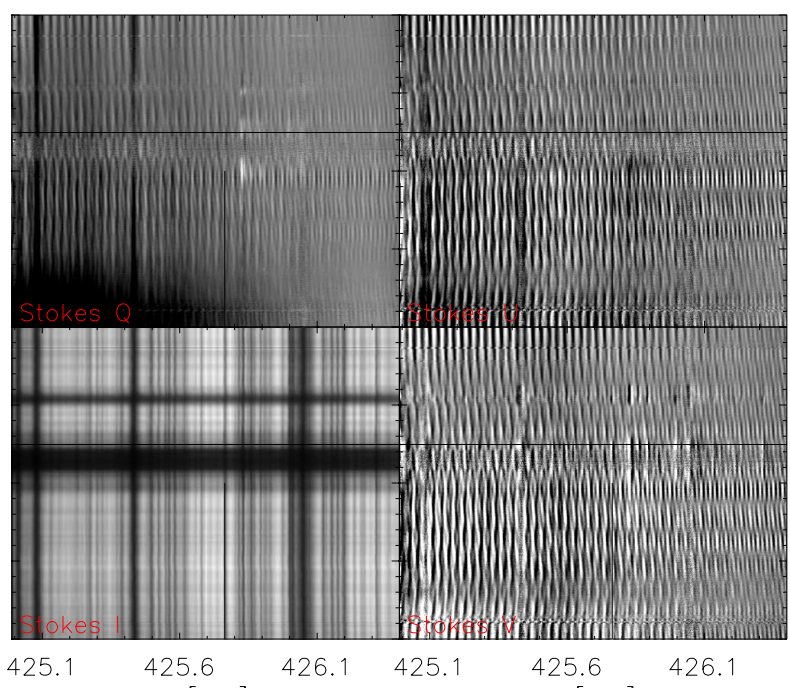

$$
\lambda[\mathrm{nm}] \quad \lambda[\mathrm{nm}]
$$

Fig. A.3. Observed example spectra of Mn I at $425.8 \mathrm{~nm}$. Left four panels: individual IQUV profiles from the location indicated with a horizontal black line in the right panels. The red line shows the corresponding profile from the FTS atlas. Right four panels: slit spectra of (clockwise, starting left bottom) IQUV on a cut across the center of a sunspot. The line without LP is indicated by a black vertical bar. 

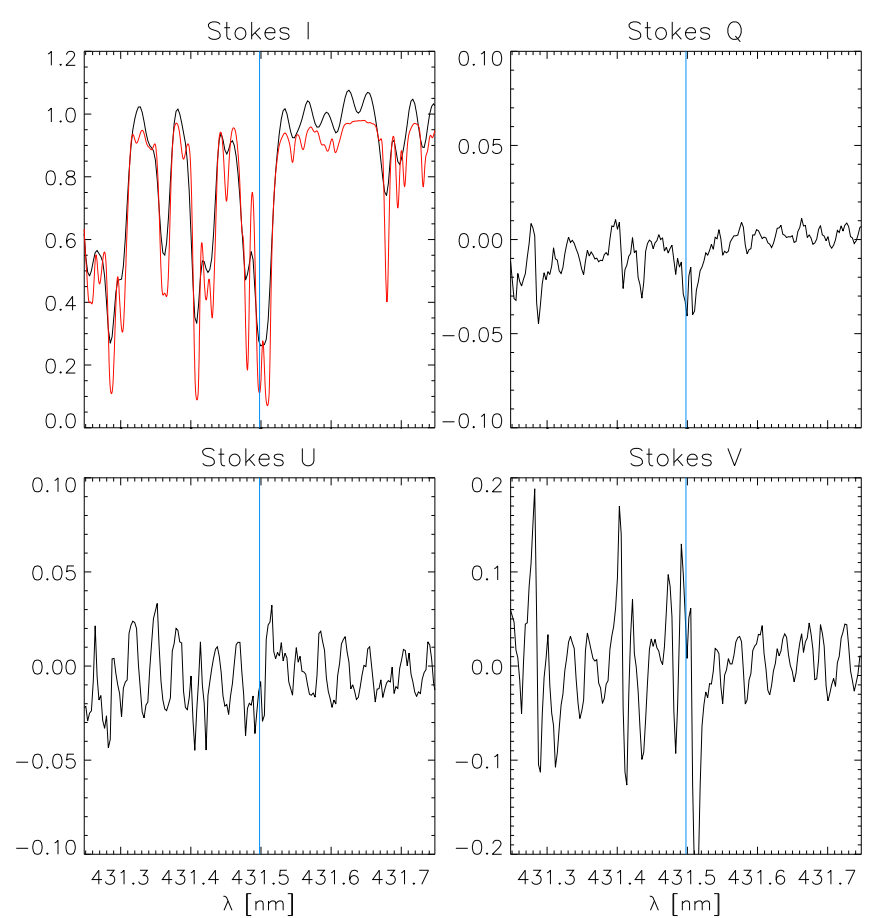

Fig. A.4. As in Fig. A.3 but for Ti II at $431.5 \mathrm{~nm}$.
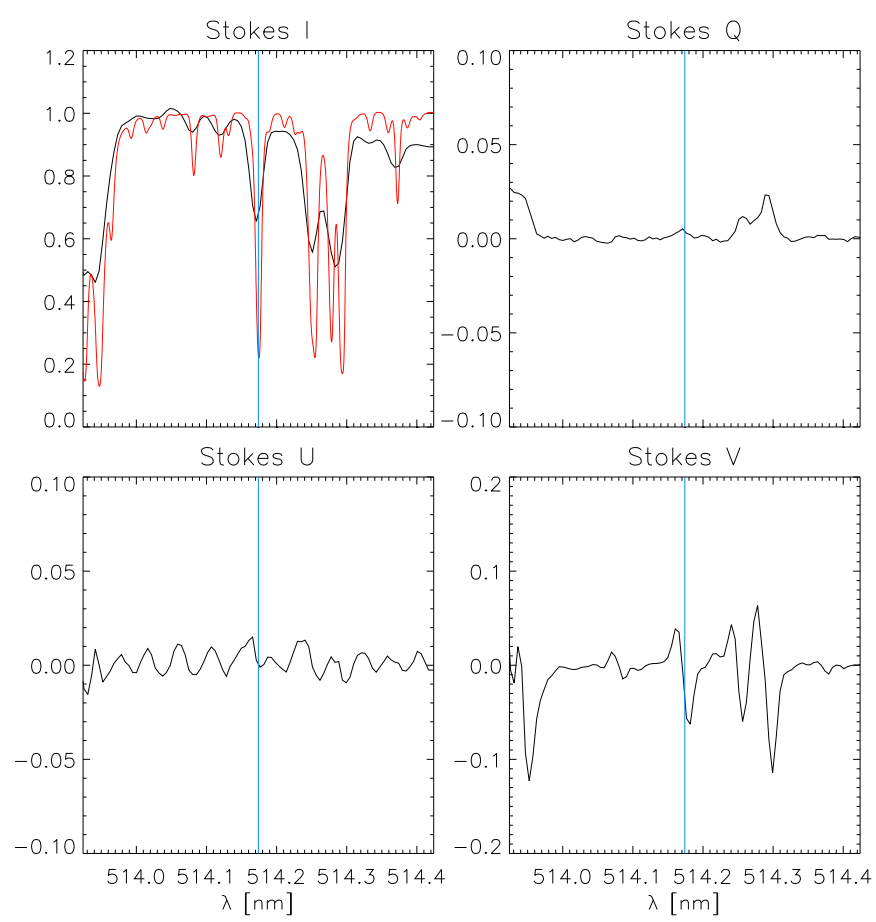

Fig. A.5. Same as Fig. A.3 for Fe I at 514.2 nm.
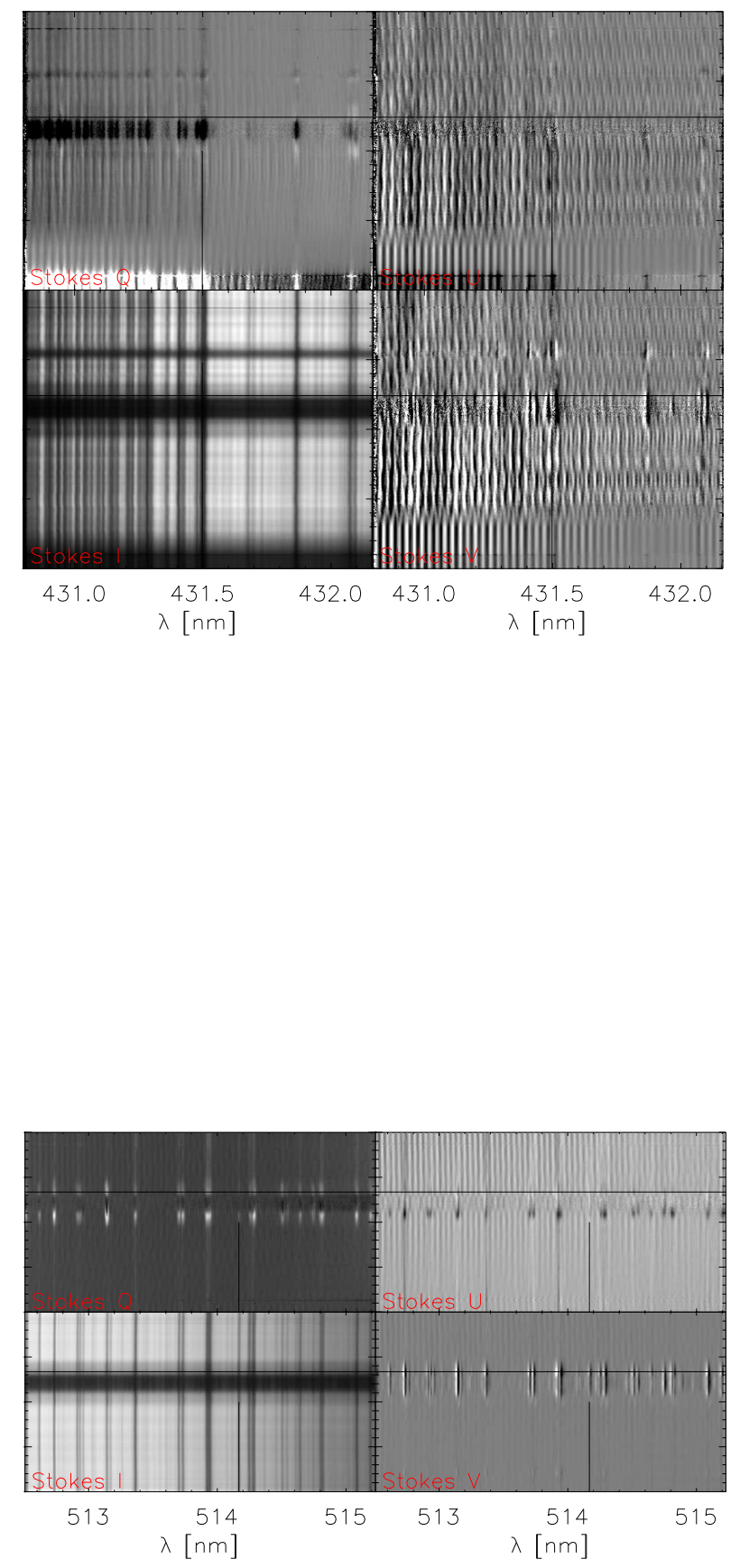


\section{Appendix B: Transition parameters and polarization amplitudes}

Tables B.1 and B. 2 contain the transition parameters and polarization amplitudes from the synthesis with $1500 \mathrm{G}$ for all lines without and with small intrinsic linear polarization, respectively. All spectral lines for which the maximal Stokes $V$ amplitude in the $1500 \mathrm{G}$ synthesis is about $8 \%$ or more are suitable for the inference of telescope properties as demonstrated with the Ca I line at $526.2 \mathrm{~nm}$. For lines with lower polarization amplitudes, the problem reduces to the question whether the crosstalk is large enough for the spurious signals to still be measured in the observations. For the case of the DST, the $V \rightarrow Q U$ crosstalk can be huge with values of $T_{i 4} / T_{44}$ above 1 , but for other telescopes that number can be as small as a few percent. This implies that polarization signals at the $10^{-4}-10^{-3}$ level have to be measured.
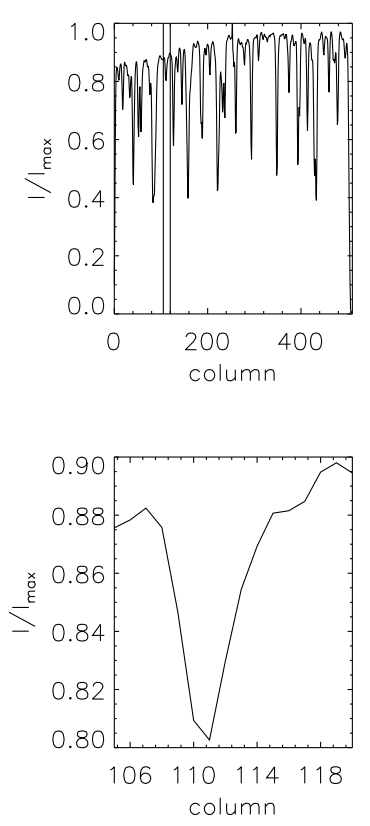
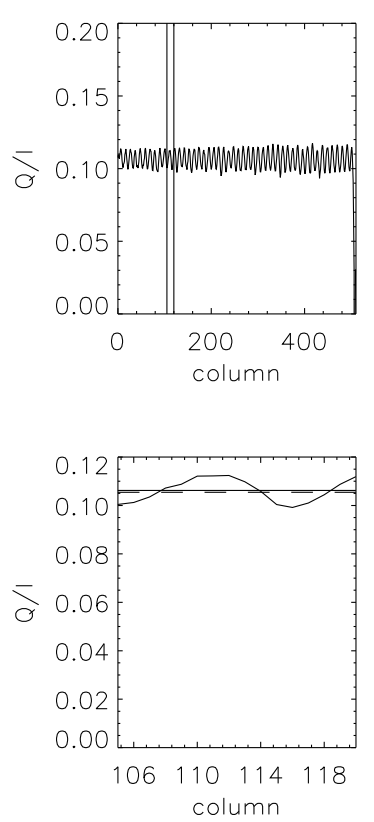
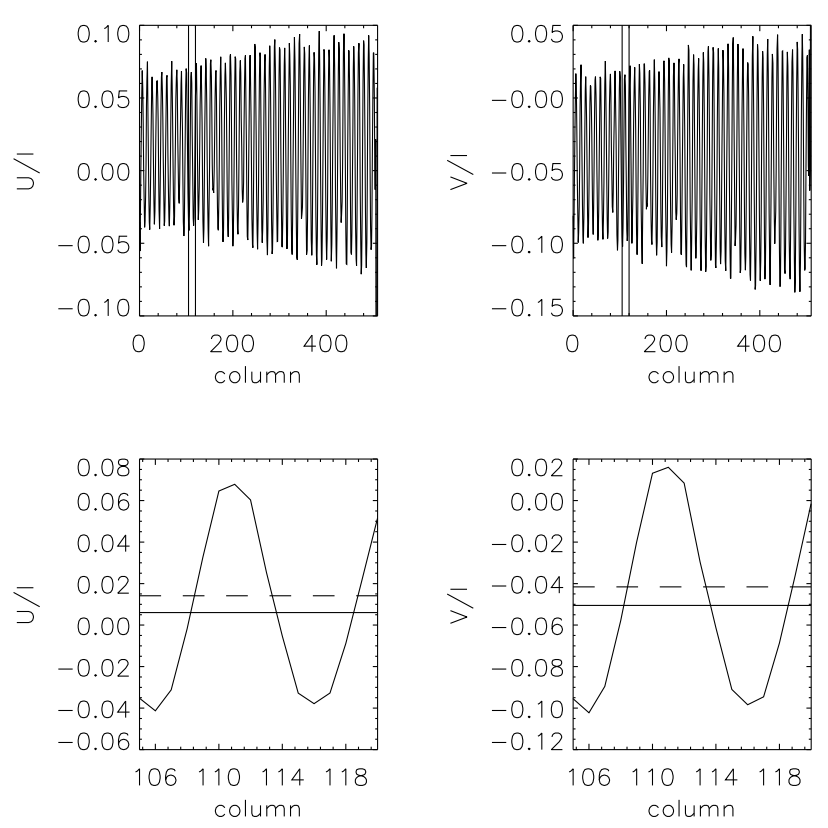

Fig. B.1. Determination of the $I \rightarrow Q U V$ crosstalk at $459 \mathrm{~nm}$. Left to right: Stokes $I, Q / I, U / I$, and $V / I$ for a single, randomly picked profile. Top row: full spectrum. The two vertical lines indicate the location of the continuum wavelength window used in the determination of $I \rightarrow Q U V$. Bottom row: spectrum inside the continuum wavelength window. The horizontal solid and dashed lines indicate the average value in the continuum window and the full spectrum, respectively.
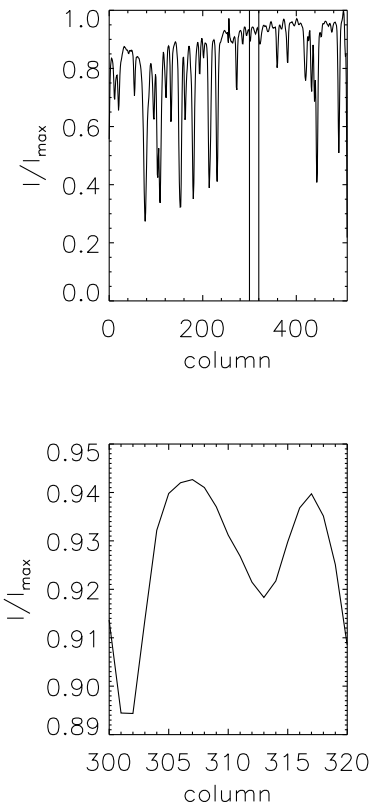
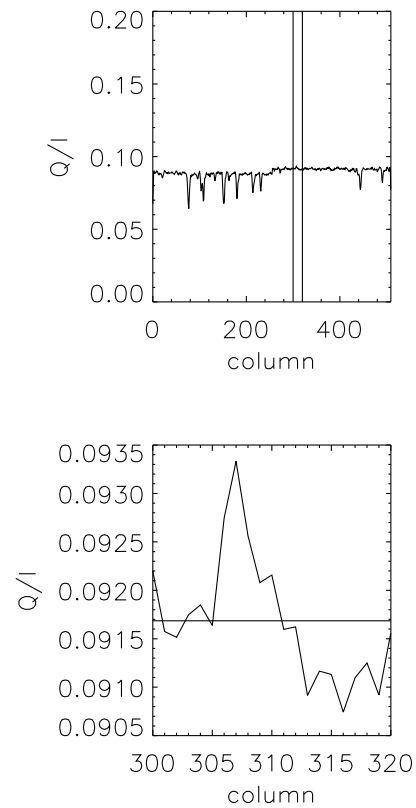
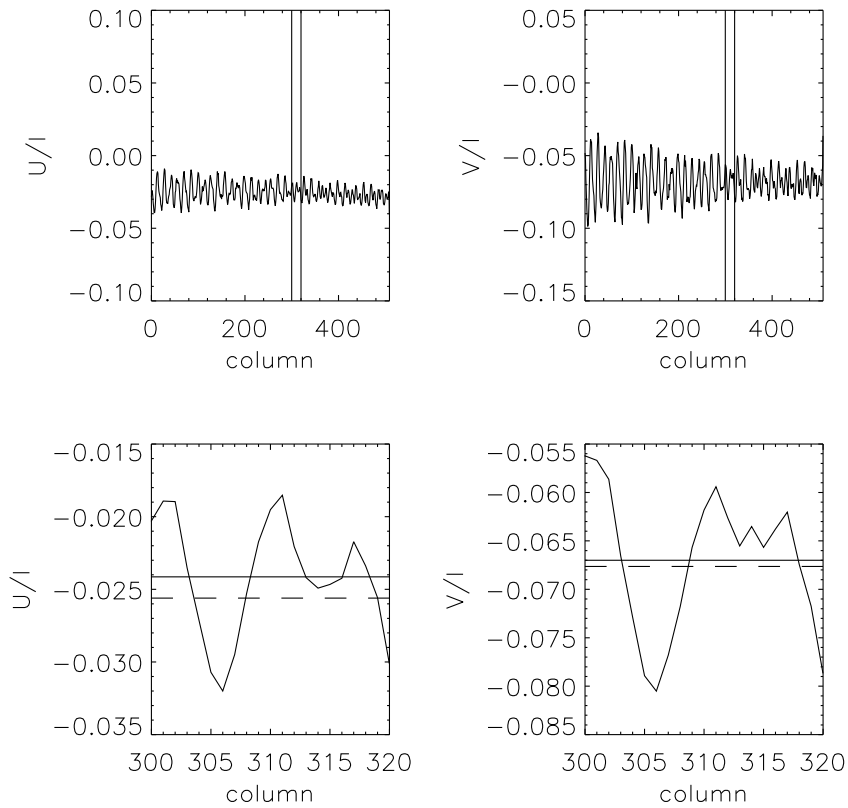

column

Fig. B.2. As in Fig. B.1 but at $526 \mathrm{~nm}$. 
Table B.1. Spectral lines without linear polarization.

\begin{tabular}{|c|c|c|c|c|c|c|c|c|}
\hline Element & $\begin{array}{l}\text { Ion. } \\
\text { state }\end{array}$ & $\begin{array}{c}\lambda_{0} \\
{[\AA]} \\
\end{array}$ & $\begin{array}{c}\text { Excitation } \\
\text { potential }(\mathrm{eV})\end{array}$ & $\log (g f)$ & Transition & $g_{\mathrm{eff}}$ & $\min . I$ & $\max .|V / I|$ \\
\hline $\mathrm{Cr}$ & II & 4087.59 & 3.103 & -3.22 & 4D $0.5-6 \mathrm{D} 0.5$ & 1.667 & 0.77028 & 0.0090 \\
\hline $\mathrm{V}$ & $\mathrm{I}$ & 4093.5 & 1.183 & -1.02 & 4P $0.5-4 \mathrm{D} 0.5$ & 1.333 & 0.92144 & 0.0028 \\
\hline V & I & 4153.32 & 0.262 & -2.55 & 6D $0.5-4 \mathrm{D} 0.5$ & 1.667 & 0.98063 & 0.0009 \\
\hline $\mathrm{Cu}$ & I & 4248.95 & 5.076 & -0.976 & 4P $0.5-4 \mathrm{D} 0.5$ & 1.333 & 0.98631 & 0.0005 \\
\hline Mn & I & 4257.66 & 2.953 & -0.70 & 4D $0.5-4 \mathrm{P} 0.5$ & 1.333 & 0.18989 & 0.0277 \\
\hline $\mathrm{S}$ & II & 4278.5 & 16.092 & -0.11 & 4P $0.5-4 \mathrm{D} 0.5$ & 1.333 & 1.00043 & $2.1 \mathrm{e}-8$ \\
\hline $\mathrm{Ti}$ & II & 4314.9 & 1.1609 & -1.104 & 4P $0.5-4 \mathrm{D} 0.5$ & 1.333 & 0.07868 & 0.0227 \\
\hline $\mathrm{Fe}$ & II & 4385.38 & 2.778 & -2.6 & 4P $0.5-4 \mathrm{D} 0.5$ & 1.333 & 0.12297 & 0.0216 \\
\hline $\mathrm{Ti}$ & II & 4407.67 & 1.221 & -2.617 & 2P $0.5-4 \mathrm{D} 0.5$ & 0.333 & 0.37181 & 0.0050 \\
\hline $\mathrm{Ti}$ & II & 4411.93 & 1.224 & -2.524 & 4P $0.5-4 \mathrm{D} 0.5$ & 1.333 & 0.32543 & 0.0215 \\
\hline $\mathrm{S}$ & II & 4456.38 & 15.848 & -0.55 & 4D $0.5-4 \mathrm{P} 0.5$ & 1.333 & 1.00015 & $9.5 e-09$ \\
\hline $\mathrm{Cr}$ & II & 4588.20 & 4.071 & -0.64 & 4D $0.5-6 \mathrm{~F} 0.5$ & 0.333 & 0.18781 & 0.0054 \\
\hline $\mathrm{V}$ & I & 4626.48 & 1.043 & -1.24 & 4D $0.5-4 \mathrm{P} 0.5$ & 1.333 & 0.93216 & 0.0026 \\
\hline V & I & 4757.48 & 2.029 & -0.29 & $6 \mathrm{G} 1.5-6 \mathrm{~F} 0.5$ & 0.167 & 0.93489 & 0.0003 \\
\hline Co & I & 5247.93 & 1.785 & -2.08 & 4P $0.5-4 \mathrm{D} 0.5$ & 1.333 & 0.75913 & 0.0112 \\
\hline Mn & I & 5292.87 & 3.383 & -2.90 & 4P $0.5-4 \mathrm{D} 0.5$ & 1.333 & 0.99262 & 0.0003 \\
\hline S & II & 5473.62 & 13.584 & -0.226 & 4P $0.5-4 \mathrm{D} 0.5$ & 1.333 & 1.00030 & $3.3 \mathrm{e}-07$ \\
\hline $\mathrm{C}$ & II & 5818.31 & 22.528 & -1.464 & 4D $0.5-4 \mathrm{P} 0.5$ & 1.333 & 0.99982 & $7.6 e-17$ \\
\hline $\mathrm{V}$ & I & 6008.67 & 1.183 & -2.34 & 4P $0.5-4 \mathrm{D} 0.5$ & 1.333 & 0.99551 & 0.0002 \\
\hline $\mathrm{V}$ & I & 6111.67 & 1.043 & -0.715 & 4D $0.5-4 \mathrm{P} 0.5$ & 1.333 & 0.80069 & 0.0101 \\
\hline $\mathrm{Co}$ & I & 6117.00 & 1.785 & -2.49 & 4P $0.5-4 \mathrm{D} 0.5$ & 1.333 & 0.89543 & 0.0058 \\
\hline $\mathrm{Fe}$ & II & 6149.23 & 3.889 & -2.8 & 4D $0.5-4 \mathrm{P} 0.5$ & 1.333 & 0.54384 & 0.0186 \\
\hline $\mathrm{S}$ & II & 6397.99 & 14.154 & -0.791 & 4D $0.5-4 \mathrm{P} 0.5$ & 1.333 & 0.99974 & $2.3 e-08$ \\
\hline $\mathrm{N}$ & I & 6646.50 & 11.750 & -1.539 & 4D $0.5-4 \mathrm{P} 0.5$ & 1.333 & 1.00055 & $4.2 \mathrm{e}-06$ \\
\hline $\mathrm{C}$ & II & 6787.21 & 20.701 & -0.377 & 4P $0.5-4 \mathrm{D} 0.5$ & 1.333 & 1.00038 & $7.7 \mathrm{e}-12$ \\
\hline $\mathrm{F}$ & I & 6870.22 & 12.751 & -0.27 & 4P $0.5-4 \mathrm{D} 0.5$ & 1.333 & 1.00011 & $5.3 e-09$ \\
\hline $\mathrm{Co}$ & I & 6872.40 & 2.008 & -1.85 & 4P $0.5-4 \mathrm{D} 0.5$ & 1.333 & 0.76954 & 0.0141 \\
\hline $\mathrm{Cl}$ & I & 8428.25 & 9.029 & -0.29 & 4P $0.5-4 \mathrm{D} 0.5$ & 1.333 & 0.99912 & $6.9 \mathrm{e}-05$ \\
\hline $\mathrm{N}$ & I & 8703.25 & 10.326 & -0.310 & 4P $0.5-4 \mathrm{D} 0.5$ & 1.333 & 0.97682 & 0.0009 \\
\hline $\mathrm{P}$ & I & 10596.90 & 6.935 & -0.24 & 4P $0.5-4 \mathrm{D} 0.5$ & 1.333 & 0.94471 & 0.0033 \\
\hline $\mathrm{N}$ & I & 11294.26 & 11.750 & -0.531 & 4D $0.5-4 \mathrm{P} 0.5$ & 1.333 & 0.99809 & $7.5 \mathrm{e}-05$ \\
\hline
\end{tabular}

Notes. Transition parameters, minimal line-core intensity, and maximal Stokes $V$ polarization amplitudes at $1500 \mathrm{G}$.
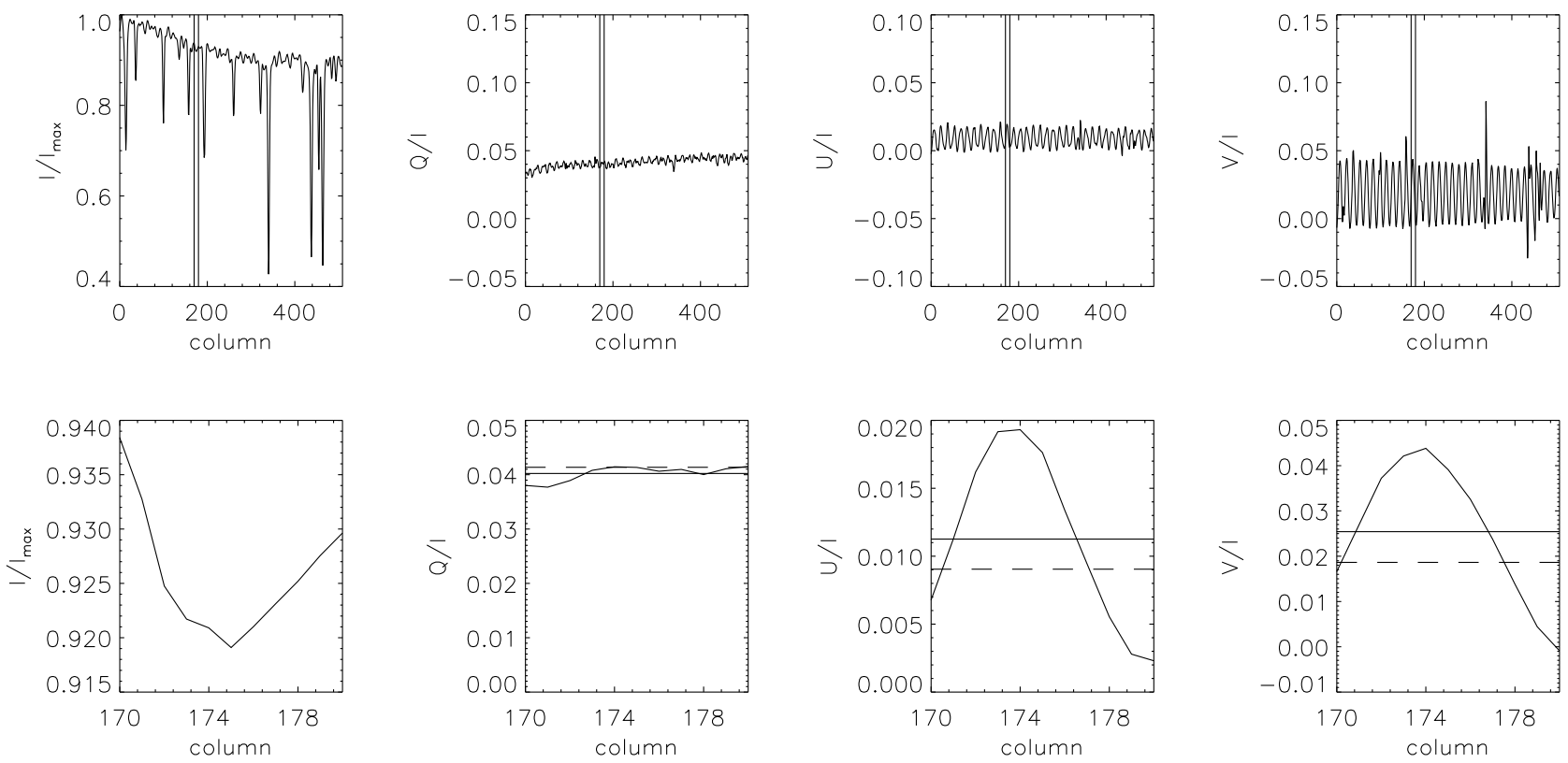

Fig. B.3. As in Fig. B.1 but at $615 \mathrm{~nm}$. 
Table B.2. Spectral lines with small linear polarization.

\begin{tabular}{|c|c|c|c|c|c|c|c|c|c|}
\hline Elem. & $\begin{array}{l}\text { Ion. } \\
\text { state }\end{array}$ & $\begin{array}{c}\lambda_{0} \\
{[\AA]}\end{array}$ & $\begin{array}{c}\text { Excit. } \\
\text { pot. }(\mathrm{eV})\end{array}$ & $\log (g f)$ & Transition & $g_{\mathrm{eff}}$ & $\max .|Q / I|$ & $\max .|U / I|$ & $\max .|V / I|$ \\
\hline $\mathrm{Ti}$ & I & 4005.95 & 2.103 & -0.53 & $5 \mathrm{~F} 3.0-5 \mathrm{H} 4.0$ & 0.375 & 0.0002 & $2.7 e-06$ & 0.0085 \\
\hline $\mathrm{Fe}$ & I & 4007.27 & 2.759 & -1.276 & $3 \mathrm{G} 3.0-3 \mathrm{~F} 2.0$ & 0.500 & 0.0249 & 0.0147 & 0.0874 \\
\hline $\mathrm{Fe}$ & I & 4017.08 & 2.759 & -1.992 & $3 \mathrm{G} 3.0-3 \mathrm{D} 2.0$ & 0.333 & 0.0026 & 0.0003 & 0.0398 \\
\hline $\mathrm{He}$ & I & 4026.19 & 20.96 & -1.453 & 3P $1.0-3 \mathrm{D} 1.0$ & 1.000 & $2.3 e-09$ & $8.1 e-15$ & $8.0 \mathrm{e}-08$ \\
\hline $\mathrm{Fe}$ & I & 4076.22 & 3.071 & -1.99 & 3P $1.0-3 \mathrm{D} 1.0$ & 1.000 & 0.0084 & 0.0020 & 0.0896 \\
\hline $\mathrm{Fe}$ & I & 4109.80 & 2.845 & -0.940 & 3P $1.0-3 \mathrm{D} 1.0$ & 1.000 & 0.0056 & 0.0064 & 0.0738 \\
\hline $\mathrm{Co}$ & I & 4132.14 & 1.049 & -2.82 & 2F $2.5-4 \mathrm{D} 2.5$ & 1.114 & 0.0012 & $3.9 \mathrm{e}-05$ & 0.0217 \\
\hline $\mathrm{Al}$ & II & 4227.95 & 15.062 & -1.709 & 3D 2.0-3F 2.0 & 0.917 & $1.5 e-09$ & $7.8 e-16$ & $2.4 \mathrm{e}-08$ \\
\hline $\mathrm{Fe}$ & I & 4229.51 & 3.274 & -1.628 & 3D 1.0-3P 1.0 & 1.000 & 0.0106 & 0.0029 & 0.0906 \\
\hline $\mathrm{Cr}$ & I & 4232.23 & 4.207 & -0.50 & $3 \mathrm{D} 2.0-3 \mathrm{G} 3.0$ & 0.333 & $9.8 \mathrm{e}-05$ & $7.5 e-07$ & 0.0036 \\
\hline $\mathrm{Fe}$ & II & 4296.57 & 2.704 & -2.9 & 4P $1.5-4 \mathrm{~F} 2.5$ & 0.500 & 0.0071 & 0.0016 & 0.0519 \\
\hline $\mathrm{Cl}$ & II & 4304.04 & 15.712 & -0.68 & 3D 1.0-3P 1.0 & 1.000 & $2.5 e-12$ & $1.1 \mathrm{e}-20$ & $4.5 e-11$ \\
\hline Sc & II & 4305.71 & 0.595 & -1.30 & $3 \mathrm{~F} 2.0-3 \mathrm{D} 2.0$ & 0.917 & 0.0066 & 0.0013 & 0.0806 \\
\hline $\mathrm{Cr}$ & I & 4312.47 & 3.113 & -1.37 & $3 \mathrm{~F} 3.0-3 \mathrm{H} 4.0$ & 0.375 & 0.0003 & $4.5 e-06$ & 0.0055 \\
\hline $\mathrm{Fe}$ & I & 4367.90 & 1.608 & -2.886 & $3 \mathrm{~F} 2.0-5 \mathrm{G} 2.0$ & 0.500 & 0.0004 & 0.0002 & 0.0554 \\
\hline $\mathrm{Cr}$ & I & 4410.96 & 2.983 & -1.22 & $3 \mathrm{H} 5.0-5 \mathrm{~F} 4.0$ & 0.400 & 0.0001 & $2.9 \mathrm{e}-06$ & 0.0097 \\
\hline $\mathrm{Fe}$ & I & 4422.57 & 2.845 & -1.115 & 3P $1.0-3 \mathrm{D} 1.0$ & 1.000 & 0.0085 & 0.0082 & 0.0784 \\
\hline $\mathrm{Cr}$ & I & 4429.92 & 3.556 & -0.67 & 3D $1.0-3 \mathrm{P} 1.0$ & 1.000 & 0.0013 & $6.8 \mathrm{e}-05$ & 0.0245 \\
\hline $\mathrm{Ca}$ & I & 4435.69 & 1.886 & -0.519 & 3P $1.0-3 \mathrm{D} 1.0$ & 1.000 & 0.0078 & 0.0064 & 0.0735 \\
\hline $\mathrm{He}$ & I & 4471.48 & 20.964 & -1.036 & 3P $1.0-3 \mathrm{D} 2.0$ & 1.000 & $1.9 \mathrm{e}-08$ & $1.4 \mathrm{e}-13$ & $1.4 \mathrm{e}-07$ \\
\hline $\mathrm{Ni}$ & I & 4513.0 & 3.706 & -1.47 & 3D 2.0-3F 2.0 & 0.917 & 0.0021 & 0.0002 & 0.0337 \\
\hline $\mathrm{Cr}$ & II & 4539.59 & 4.0423 & -2.53 & $2 \mathrm{~F} 2.5-4 \mathrm{D} 2.5$ & 1.114 & 0.0009 & $3.2 \mathrm{e}-05$ & 0.0168 \\
\hline $\mathrm{Al}$ & II & 4589.67 & 15.062 & -1.608 & 3D 2.0-3F 2.0 & 0.917 & $1.5 e-09$ & $9.0 e-16$ & $2.3 e-08$ \\
\hline $\mathrm{P}$ & II & 4626.70 & 12.812 & -0.32 & 3D 2.0-3F 2.0 & 0.917 & $6.5 e-09$ & $1.5 \mathrm{e}-14$ & $9.6 e-08$ \\
\hline $\mathrm{Cr}$ & I & 4698.94 & 3.079 & -1.44 & $3 \mathrm{G} 3.0-3 \mathrm{D} 2.0$ & 0.333 & 0.0001 & $1.1 \mathrm{e}-06$ & 0.0047 \\
\hline $\mathrm{Ti}$ & I & 4722.61 & 1.053 & -1.33 & 3P $1.0-3 \mathrm{D} 1.0$ & 1.000 & 0.0020 & 0.0001 & 0.0380 \\
\hline $\mathrm{C}$ & I & 4738.21 & 7.946 & -3.115 & 3D $1.0-3 \mathrm{P} 1.0$ & 1.000 & $7.0 \mathrm{e}-05$ & $3.4 \mathrm{e}-07$ & 0.0014 \\
\hline $\mathrm{Cr}$ & I & 4767.27 & 3.556 & -1.02 & 3D 2.0-3F 2.0 & 0.917 & 0.0008 & $2.0 \mathrm{e}-05$ & 0.0115 \\
\hline $\mathrm{Cl}$ & II & 4778.91 & 17.086 & -0.35 & $3 \mathrm{P} 1.0-3 \mathrm{D} 1.0$ & 1.000 & $4.5 e-13$ & $5.6 e-22$ & $7.7 e-12$ \\
\hline $\mathrm{Ni}$ & $\mathrm{I}$ & 4808.87 & 3.706 & -1.41 & 3D $2.0-3 \mathrm{G} 3.0$ & 0.333 & 0.0003 & $1.2 \mathrm{e}-05$ & 0.0151 \\
\hline $\mathrm{Fe}$ & I & 4813.11 & 3.274 & -2.84 & 3D $1.0-5 \mathrm{D} 1.0$ & 1.000 & 0.0020 & 0.0002 & 0.0399 \\
\hline $\mathrm{Si}$ & I & 4823.32 & 4.930 & -2.33 & 3P $1.0-3 \mathrm{D} 1.0$ & 1.000 & 0.0016 & 0.0001 & 0.0315 \\
\hline $\mathrm{Fe}$ & II & 4833.19 & 2.657 & -4.8 & $4 \mathrm{H} 5.5-6 \mathrm{~F} 4.5$ & 0.455 & 0.0002 & $2.7 e-06$ & 0.0079 \\
\hline $\mathrm{Sr}$ & I & 4876.08 & 1.798 & -0.551 & 3P $1.0-3 \mathrm{D} 1.0$ & 1.000 & $7.8 \mathrm{e}-05$ & $1.8 \mathrm{e}-07$ & 0.0014 \\
\hline $\mathrm{Cl}$ & II & 4922.15 & 15.714 & -0.59 & 3D 2.0-3F 2.0 & 0.917 & $1.9 \mathrm{e}-12$ & $7.3 e-21$ & $2.7 e-11$ \\
\hline $\mathrm{Cl}$ & II & 4924.25 & 15.626 & -1.54 & $3 \mathrm{~F} 2.0-3 \mathrm{D} 2.0$ & 0.917 & $2.5 e-13$ & $1.2 \mathrm{e}-22$ & $3.4 \mathrm{e}-12$ \\
\hline $\mathrm{P}$ & II & 4927.20 & 12.791 & -0.68 & 3D 1.0-3P 1.0 & 1.000 & $2.3 e-09$ & $2.4 \mathrm{e}-15$ & $3.8 \mathrm{e}-08$ \\
\hline $\mathrm{Fe}$ & $\mathrm{I}$ & 4930.32 & 3.960 & -1.201 & 3D $1.0-5 \mathrm{D} 1.0$ & 1.000 & 0.0097 & 0.0025 & 0.0875 \\
\hline $\mathrm{Cr}$ & I & 4936.34 & 3.113 & -0.34 & $3 \mathrm{~F} 3.0-3 \mathrm{H} 4.0$ & 0.375 & 0.0018 & 0.0002 & 0.0317 \\
\hline $\mathrm{Ti}$ & I & 4941.57 & 2.160 & -1.01 & 3D 2.0-3F 2.0 & 0.917 & 0.0005 & $7.5 e-06$ & 0.0075 \\
\hline $\mathrm{Ti}$ & I & 4997.09 & 0.000 & -2.056 & $3 \mathrm{~F} 2.0-3 \mathrm{D} 2.0$ & 0.917 & 0.0035 & 0.0005 & 0.0620 \\
\hline $\mathrm{Fe}$ & I & 5021.59 & 4.256 & -0.677 & $5 \mathrm{~F} 3.0-5 \mathrm{H} 4.0$ & 0.375 & 0.0036 & 0.0005 & 0.0441 \\
\hline $\mathrm{Fe}$ & I & 5099.08 & 3.984 & -1.265 & $3 \mathrm{~F} 2.0-3 \mathrm{D} 2.0$ & 0.917 & 0.0092 & 0.0021 & 0.0830 \\
\hline $\mathrm{Fe}$ & I & 5141.74 & 2.424 & -2.238 & 3P $1.0-3 \mathrm{D} 1.0$ & 1.000 & 0.0162 & 0.0069 & 0.0916 \\
\hline $\mathrm{Fe}$ & I & 5207.94 & 3.635 & -2.40 & 3D $1.0-3 \mathrm{P} 1.0$ & 1.000 & 0.0022 & 0.0003 & 0.0465 \\
\hline $\mathrm{Ca}$ & I & 5261.71 & 2.521 & -0.73 & 3D 1.0-3P 1.0 & 1.000 & 0.0120 & 0.0039 & 0.0845 \\
\hline $\mathrm{Al}$ & II & 5280.27 & 15.586 & -1.981 & 3P $1.0-3 \mathrm{D} 1.0$ & 0.917 & $1.8 \mathrm{e}-10$ & $1.9 \mathrm{e}-17$ & $3.0 \mathrm{e}-09$ \\
\hline $\mathrm{Ti}$ & I & 5300.01 & 1.053 & -1.47 & 3P $1.0-3 \mathrm{D} 1.0$ & 1.000 & 0.0016 & $8.7 e-05$ & 0.0307 \\
\hline $\mathrm{C}$ & I & 5300.87 & 8.640 & -2.622 & 3D 1.0-3P 1.0 & 1.000 & $5.9 e-05$ & $3.0 \mathrm{e}-07$ & 0.0011 \\
\hline $\mathrm{Fe}$ & I & 5341.02 & 1.608 & -1.953 & $3 \mathrm{~F} 2.0-3 \mathrm{D} 2.0$ & 0.917 & 0.0072 & 0.0056 & 0.0778 \\
\hline $\mathrm{Ni}$ & I & 5353.39 & 1.951 & -2.81 & 3P $1.0-3 \mathrm{D} 1.0$ & 1.000 & 0.0039 & 0.0006 & 0.0670 \\
\hline $\mathrm{Ni}$ & I & 5514.79 & 3.847 & -1.99 & 1F $3.0-3 \mathrm{P} 2.0$ & 0.500 & 0.0004 & $5.2 \mathrm{e}-06$ & 0.0055 \\
\hline $\mathrm{Fe}$ & I & 5667.66 & 2.609 & -2.94 & $3 \mathrm{~F} 2.0-3 \mathrm{D} 2.0$ & 0.917 & 0.0064 & 0.0013 & 0.0790 \\
\hline $\mathrm{Fe}$ & I & 5747.95 & 4.608 & -1.41 & $3 \mathrm{~F} 3.0-3 \mathrm{H} 4.0$ & 0.375 & 0.0011 & $8.4 \mathrm{e}-05$ & 0.0212 \\
\hline $\mathrm{Fe}$ & I & 6085.26 & 2.759 & -2.712 & 3G 3.0-3D 2.0 & 0.333 & 0.0017 & 0.0002 & 0.0358 \\
\hline $\mathrm{Fe}$ & I & 6127.91 & 4.413 & -1.399 & $3 \mathrm{~F} 3.0-3 \mathrm{H} 4.0$ & 0.375 & 0.0017 & 0.0002 & 0.0285 \\
\hline
\end{tabular}

Notes. Transition parameters and maximal Stokes $Q U V / I$ polarization amplitudes at $1500 \mathrm{G}$. 


\section{Appendix C: Error estimates for measurements}

\section{C.1. Crosstalk from intensity to polarization $I \rightarrow Q U V$}

The crosstalk from intensity to polarization $I \rightarrow Q U V$ was determined as part of the 2013 data reduction pipeline prior to fringe removal. A window of continuum wavelengths close to the spectral line of interest was specified (see Figs. B.1-B.3). The extent of the window was chosen to be as large as possible without including solar spectral lines with genuine polarization signal. The $I \rightarrow Q U V$ crosstalk was then determined as the average value of $Q U V / I$ inside the continuum window.

The fringe amplitude in the spectra can reach up to several percent but generally decreases with increasing wavelength (Figs. B.1-B.3). Each time a complete fringe period is covered in the continuum wavelength window, its contribution to the average value of $Q U V / I$ is zero. The maximum effect that fringes can cause happens when half a period of the fringe pattern is not compensated by the corresponding half with opposite sign. This residual contribution scales with the fringe period relative to the total extent of the continuum window.
To estimate the possible error in the determination of the $I \rightarrow Q U V$ crosstalk introduced by the fringe pattern, we randomly picked one individual profile from one map at 459, 526, and $615 \mathrm{~nm}$. We determined the value of $I \rightarrow Q U V$ in the same continuum window as used in the data reduction and averaged it over the full wavelength range of the spectra for comparison (Table C.1). The difference between the two values is between 0.001 and 0.01 while Figs. B.1-B.3 show that the average value of $Q U V / I$ is well recovered even in the presence of fringes in individual profiles. The fringe pattern showed a variation in time, space, and wavelength, whereas the $I \rightarrow Q U V$ crosstalk values used in the fit of telescope parameters were an average of more than 100 profiles for each scan step. The error for a single value of $I \rightarrow Q U V$ in, for example, Fig. 12 should thus be $<1 \%$.

\section{C.2. Crosstalk circular to linear polarization $V \rightarrow Q U$}

It is somewhat more difficult to estimate the potential impact of the fringe pattern on the $V \rightarrow Q U$ crosstalk. The genuine polarization signal varies between individual profiles much more than the intensity crosstalk, in addition to the variable fringe pattern. Similar to the $I \rightarrow Q U V$ crosstalk, the data points for $V \rightarrow Q U$ were obtained by an average along the slit, but now over a
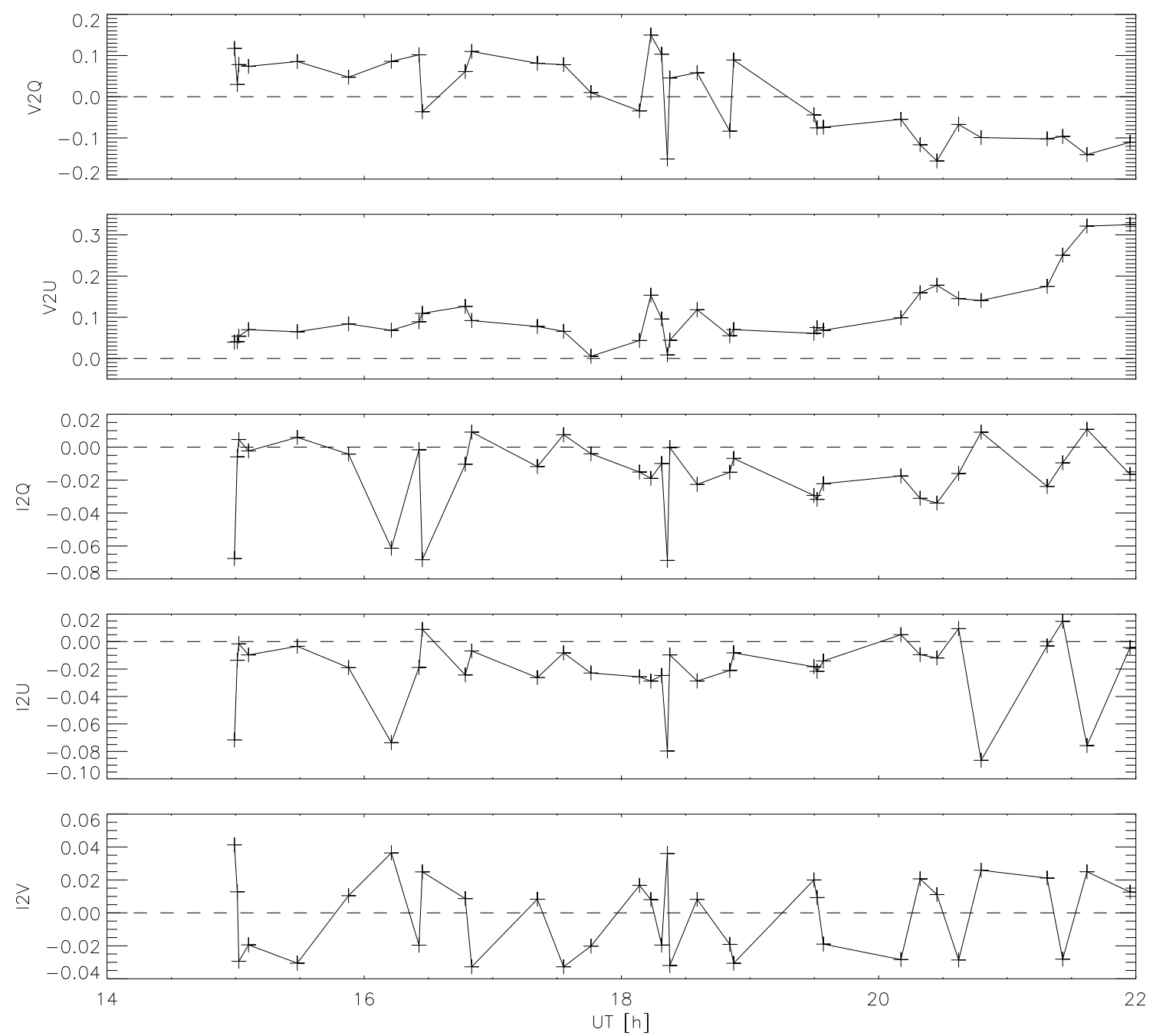

Fig. C.1. Fit residuals at $614.9 \mathrm{~nm}$. Top to bottom: $V \rightarrow Q, V \rightarrow U, I \rightarrow Q U V$. 
Table C.1. $I \rightarrow Q U V$ for individual profiles.

\begin{tabular}{ccc}
\hline \hline & $\begin{array}{c}\text { Full } \\
\text { profile }\end{array}$ & $\begin{array}{c}\text { Continuum } \\
\text { window }\end{array}$ \\
\hline$\lambda$ & \multicolumn{2}{c}{$458.8 \mathrm{~nm}$} \\
\hline$Q / I$ & 0.105 & 0.106 \\
$U / I$ & 0.014 & 0.006 \\
$V / I$ & -0.042 & -0.051 \\
\hline$\lambda$ & \multicolumn{2}{c}{$526.2 \mathrm{~nm}$} \\
\hline$Q / I$ & 0.088 & 0.092 \\
$U / I$ & -0.026 & -0.024 \\
$V / I$ & -0.068 & -0.067 \\
\hline$\lambda$ & \multicolumn{2}{c}{$614.9 \mathrm{~nm}$} \\
\hline$Q / I$ & 0.041 & 0.041 \\
$U / I$ & 0.009 & 0.011 \\
$V / I$ & 0.019 & 0.020 \\
\hline
\end{tabular}

Table C.2. Root mean square values of the fit residuals at $614.9 \mathrm{~nm}$.

\begin{tabular}{lcccc}
\hline \hline$V \rightarrow Q$ & $V \rightarrow U$ & $I \rightarrow Q$ & $I \rightarrow U$ & $I \rightarrow V$ \\
\hline 0.093 & 0.074 & 0.022 & 0.026 & 0.024 \\
\hline
\end{tabular}

variable number of pixels depending on the presence of significant polarization signal in the masks (see Fig. 7).

As an estimate of the error in the $V \rightarrow Q U$ crosstalk, we used the fit residuals at $614.9 \mathrm{~nm}$ for all quantities used in the fit (see Fig. C.1), that is, the difference between the observations and the final best-fit solution for the telescope model in $V \rightarrow Q U$ and $I \rightarrow Q U V$. The standard deviation of the residuals can be used as a first-order estimate of the random errors in the quantities although the residuals at this specific wavelength also show some more systematic variation, for example, a sort of a linear trend in $V \rightarrow Q$ and an offset from zero in $V \rightarrow U$. Table C.2 lists the rms values of the fit residuals at $614.9 \mathrm{~nm}$. The rms of the $V \rightarrow Q U$ residuals is about $8 \%$, while $I \rightarrow Q U V$ fluctuates by about $2 \%$. The error of individual data points in $V \rightarrow Q U$ used in the fit thus should be about $5 \%$ when one considers the contribution of the systematic effects to the fluctuation around the mean value.

As a last generic error estimate, we used the value of the $\chi^{2}$ at $614.9 \mathrm{~nm}$. If the reduced $\chi^{2}$ is assumed to follow a $\chi^{2}$ distribution, its value should be

$\chi_{\text {reduced }}^{2}=\frac{\chi^{2}}{N-f} \cdot \frac{1}{\sigma^{2}} \equiv 1$,

where $\chi^{2}=\sum_{i}\left(\mathrm{OBS}_{i}-\mathrm{FIT}_{i}\right)^{2}, N$ is the number of data points, $f$ is the number of degrees of freedom in the fit, and $\sigma$ is the error of one data point.

With $\chi^{2}(614.9 \mathrm{~nm})=4.808, N=5 \cdot 34$, and $f=5$, one obtains $\sigma=0.17$. This number is about twice as large as the previous estimates for $V \rightarrow Q U$, which presumably indicates that the assumption of a well-behaved $\chi^{2}$ distribution is not fully valid. 